\title{
Quasinormal Modes, Local Density of States, and Classical Purcell Factors for Coupled Loss-Gain Resonators
}

\author{
Juanjuan Ren $\odot,{ }^{1, *}$ Sebastian Franke $\odot^{2,1, \dagger}$ and Stephen Hughes $\odot^{1, *}$ \\ ${ }^{1}$ Department of Physics, Engineering Physics, and Astronomy, \\ Queen's University, Kingston, Ontario K7L 3N6, Canada \\ ${ }^{2}$ Technische Universität Berlin, Institut für Theoretische Physik, \\ Nichtlineare Optik und Quantenelektronik, Hardenbergstraße 36, 10623 Berlin, Germany
}

(Received 18 January 2021; revised 31 May 2021; accepted 17 August 2021; published 28 October 2021)

\begin{abstract}
We present a quasinormal-mode (QNM) theory for coupled loss and gain resonators working in the vicinity of an exceptional point. Assuming linear media, which can be fully quantified using the complex pole properties of the QNMs, we show how the QNMs yield a quantitatively accurate model to a full classical dipole spontaneous-emission response in Maxwell's equations at a variety of spatial positions and frequencies (under linear response). We also develop an intuitive QNM coupled-mode theory, which can be used to accurately model such systems using only the QNMs of the bare resonators, where the hybrid QNMs of the complete system are automatically obtained. Near a lossy exceptional point, whose general properties are broadened and corrected through use of QNM theory, we analytically show how the QNMs yield a Lorentzian-like and a Lorentzian-squared-like response for the spontaneous-emission line shape consistent with other works. However, using rigorous analytical and numerical solutions for microdisk resonators, we demonstrate that the general line shapes are far richer than what has been previously predicted. Indeed, the classical picture of spontaneous emission can take on a wide range of positive and negative Purcell factors from the hybrid modes of the coupled loss-gain system. The negative Purcell factors are unphysical and signal a clear breakdown of the classical dipole picture of spontaneous emission in such media, though the concept of a negative local density of states is correct. This finding has enabled a quantum fix to the decay of a two-level-system dipole emitter in amplifying and lossy media [Franke et al., Phys. Rev. Lett. 127, 013602 (2021)], and we further show and discuss the impact of this fix using the QNMs of the microdisk resonators. We also show the rich spectral features of the Green's function propagators, which can be used to model various physical observables, such as photon detection.
\end{abstract}

DOI: 10.1103/PhysRevX.11.041020

Subject Areas: Photonics

\section{INTRODUCTION}

Lossless photonic systems (such as closed resonators with no material absorption) can be formulated as a Hermitian eigenvalue problem, which yields real eigenfrequencies from the source-free Helmholz equation, and corresponding normal modes (NMs). This is also true for periodic systems with bound modes, e.g., lossless waveguide modes. However, real cavity structures (resonators) with open boundary conditions yield finite loss (or gain) and produce complex eigenfrequencies. Thus, most optical

\footnotetext{
*jr180@queensu.ca

†sebastian.r.franke@gmail.com

*shughes@queensu.ca
}

Published by the American Physical Society under the terms of the Creative Commons Attribution 4.0 International license. Further distribution of this work must maintain attribution to the author(s) and the published article's title, journal citation, and DOI. systems are naturally dissipative via material absorption and/or radiation.

A common design approach to improving resonators is to increase the photonic local density of states (LDOS) by reducing radiation losses and the effective mode volume, thus increasing the Purcell factor for enhanced spontaneous emission (SE). An alternative approach to reducing loss is through gain compensation, where a gain medium can be introduced and controlled through stimulated emission or parametric processes. Not necessarily related to lasing, a gain medium can be introduced to the system and treated as a linear amplifying medium [1,2], which must satisfy strict criteria that are quantifiable through the complex poles of the (photonic) Green's function. Aided by the rapid development of optical nanotechnologies, coupled loss and gain structures have been under intense investigation recently, especially after the demonstrations of parity-time (PT) symmetry [3-10] in optical systems [11-27], which support so-called exceptional points (EPs) [28-35] (where two isolated eigenfrequencies and eigenmodes coalesce), 
along with many interesting and counterintuitive phenomena, including nonreciprocal propagation (isolators) [36-40], mode switching [41-44], efficient sensing [45,46], absorbers [47-50], and lasing [51-54].

To have a detailed understanding of such systems, and also for connecting to new applications in quantum optics, it is desirable to have an accurate model of coupled loss-gain resonators at the level of a rigorous and intuitive mode theory, which can allow one to describe light-matter interactions at various spatial points and frequencies. From a theoretical perspective, temporal coupled-mode theory (CMT) has proven to be an efficient approach to investigate coupledresonator systems $[55,56]$, where only the solution from the bare systems is required, and one assumes that the coupled modes can be represented by a superposition of the bare modes. However, the coupling coefficients are typically used as heuristic parameters in that they are usually extracted from fitting the full solution of the coupled system properties $[38,57-59]$, or they are mainly used to explain the basic physics of coupling.

Another potential problem with such approaches is that the underlying modes of the bare resonators are assumed to be NMs (Hermitian system), and a finite decay rate to account for real losses is added phenomenologically. This finite decay rate already relates to a non-Hermitian problem, so it is natural to model the bare resonators also with a non-Hermitian theory, where the correct eigenvalues and modes would then be obtained for a more general CMT for loss-gain systems. This is not only a more correct approach, but it changes some of the fundamental coupling regimes and constraints significantly, and opens up much richer light-matter interaction regimes.

In recent years, the theory of open cavity modes has been shown to be accurately described in terms of quasinormal modes (QNMs) [60-72], which are open cavity modes with complex eigenfrequencies and spatially diverging modes (with finite loss). These open cavity modes naturally include the effect of losses and can also be used to construct the photon Green's function, which describes a wide range of light-matter interactions [64-67,71-74]. Moreover, as shown recently, QNMs can be fully quantized and used to show departures from the usual NM quantum-optics theories [75-77]. Several CMT approaches based on QNMs have also been successfully developed [78-81] for coupled passive resonator systems.

In this work, we first present a QNM theory for general media containing both lossy resonators and gain resonators. We describe a rigorous and intuitive CMT based on the Green's function solution for the coupled loss-gain QNMs, where we analytically obtain the hybrid modes from only the QNMs of the bare resonators (gain or loss). We demonstrate the extremely high accuracy of the analytical theory by comparing with full numerical dipole simulations for whispering-gallery modes (WGMs) of microdisk resonators and show excellent agreement for various designs

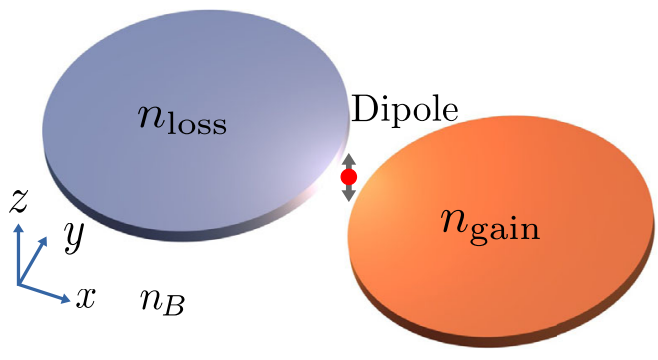

FIG. 1. Schematic diagram of a coupled loss and gain resonator system. The refractive index of the lossy (gain) resonator is $n_{\text {loss }}\left(n_{\text {gain }}\right)$, which are in a homogeneous background medium with $n_{B}$.

and spatial positions without using any fitting parameters. A QNM approach also allows one to justify the underlying assumptions of a linear gain medium, which requires an analysis of the complex poles. Without such an analysis, composite systems may not even constitute a physically meaningful solution with gain treated at the level of a linear material response in Maxwell's equations. Indeed, as far as we are aware, this is likely the only approach to confirm this directly (in the absence of having an analytical expression for the medium Green's function).

After obtaining the physically meaningful QNMs for loss-gain resonator systems, we apply our theory to study the unusual Purcell factors and Green's function propagators at various spatial positions for eigenfrequencies close to an EP. For our numerical example, the coupled loss-gain disk resonators are shown in Fig. 1(a), where one microdisk has material loss and the other has material gain. Such systems with balanced gain and loss form a general optical system to investigate PT symmetry and EP physics $[38,39,46]$. In our model, we choose a gain coefficient that is slightly less than the loss coefficient, which can support hybridized QNMs with finite loss, which is also a requirement for assuming linear gain media [1].

The rest of our paper is organized as follows: In Sec. II, we introduce optical QNMs, Green's functions in terms of QNMs, and show how these relate to SE decay and Purcell factors. In Sec. III, we present a detailed CMT using both NMs and QNMs to obtain analytical insight into coupled loss-gain resonators. We subsequently use these to explain when an EP may form and how things change when one uses a QNM theory. We obtain explicit expressions for the hybrid modes using only the QNMs of the bare loss or gain resonators. We explain the limits and failures of the usual CMT for such systems. Section IV discusses Green's functions and Purcell factors at the EP and shows how a Lorentzian-like or Lorentzian-squared-like line shape forms [82-84]. Section V presents detailed numerical results for coupled microdisk resonators and confirms the excellent agreement with our analytical CMT and full dipole solutions (namely, using a numerical solution to the full Maxwell equations with a classical dipole source) for 
various gap distances between the resonators. We then study various Purcell factor regimes at different dipole positions as a function of the frequency and show highly unusual and rich spectral line shapes, including negative Purcell factors, and discuss the essential role of the QNM phase; again, all of these show quantitatively good agreement with full dipole calculations. We stress that negative total Purcell factors are not physical and motivate the need for a corrected derivation of the accepted Fermi golden rule for such media, which is described elsewhere [85]. The role of this additional quantum fix, which can also be computed from only the properties of the hybrid QNMs, is exemplified for two selected dipole locations where the LDOS is negative and shown to yield net positive SE rates. We also show the Green's function propagators, which connects to various observables outside the resonators, which also yield rich non-Lorentzian line shapes. We give our conclusions in Sec. VI.

In addition to the main text, we also present several Appendices. Appendix A discusses the numerical QNM normalization approaches using COMSOL, where we show three different approaches yielding the same normalized QNMs within numerical precision. Appendix B discusses why we need to consider only one QNM of the microdisk resonator for the dipole locations we study, which is constructed from a symmetric linear combination of clockwise and counterclockwise WGMs. Full dipole calculations in COMSOL are also discussed in Appendix C, which are used to check the validity of the QNM results. To compare with the coupled cavity systems, the results for single-loss and single-gain cavities are shown in Appendix D, which also confirms the extremely high accuracy of the single QNM approximation for these resonators. Naturally, one can also solve the coupled system with a QNM approach directly, instead of using CMT from the bare solutions; thus, Appendix E shows the direct QNM approach for coupled resonators, where quantitatively good agreement with our analytical CMT results and full dipole results are obtained. In addition to the loss-gain cavities shown in the main text, we also show two more loss-gain examples in Appendix F, with different gain coefficients.

\section{QUASINORMAL MODES AND SEMICLASSICAL THEORY OF SPONTANEOUS EMISSION AND PURCELL FACTORS}

We first introduce the electric field QNMs $\tilde{\mathbf{f}}_{\mu}(\mathbf{r})$, which are solutions to the Helmholtz equation

$$
\boldsymbol{\nabla} \times \boldsymbol{\nabla} \times \tilde{\mathbf{f}}_{\mu}(\mathbf{r})-\left(\frac{\tilde{\omega}_{\mu}}{c}\right)^{2} \epsilon\left(\mathbf{r}, \tilde{\omega}_{\mu}\right) \tilde{\mathbf{f}}_{\mu}(\mathbf{r})=0,
$$

where $c$ is the vacuum speed of light, $\tilde{\omega}_{\mu}=\omega_{\mu}-i \gamma_{\mu}$ is the complex eigenfrequency of each QNM, and $\epsilon\left(\mathbf{r}, \tilde{\omega}_{\mu}\right)$ is the dielectric function, which is, in general, complex and dispersive, though for our numerical examples below, we assume this is a constant complex value in the frequency regime of interest (this is not a model restriction in general). The open boundary conditions ensure the Silver-Müller radiation condition [86]. It is also worth noting that this boundary condition leads to quite different asymptotic behavior of gain QNMs and loss QNMs due to the change of sign for $\gamma_{\mu}$. Namely, the lossy QNMs diverge in space but converge in time, while the gain QNMs converge in space but diverge in time. For the composite system, the hybrid modes must converge in time, forcing the complex poles to have loss. These subtleties are clearly missing and overlooked in heuristic theories of coupled loss and gain resonators but are essential to get a physically meaningful model.

Using $n_{L / G}$ to represent the real parts of the refractive index and $\alpha_{L / G}$ the loss or gain coefficients, for a lossy resonator with permittivity $\epsilon_{\text {loss }}=n_{\text {loss }}^{2} \equiv\left(n_{L}+i \alpha_{L}\right)^{2}$, we assume a dominant QNM resonance $\tilde{\omega}_{L}=\omega_{L}-i \gamma_{L}$, where $\alpha_{L}, \gamma_{L}>0$. Similarly, for the gain resonator $\epsilon_{\text {gain }}=n_{\text {gain }}^{2} \equiv\left(n_{G}+i \alpha_{G}\right)^{2}$, we have a dominant QNM resonance $\tilde{\omega}_{G}=\omega_{G}-i \gamma_{G}$, where now $\alpha_{G}, \gamma_{G}<0$. The quality factor is defined from $Q_{\mu}=\omega_{\mu} /\left(2\left|\gamma_{\mu}\right|\right)$. Since we treat the gain amplifier in terms of a linear amplifying medium (e.g., we neglect the saturation effects in the gain medium), the composite system must have $\gamma_{ \pm}>0$ for the hybrid modes [1]. We refer to the gain and loss QNMs as $\tilde{\mathbf{f}}^{G}$ and $\tilde{\mathbf{f}}^{L}$, respectively, and the hybrid modes (i.e., in the presence of coupling) as $\tilde{\mathbf{f}}^{ \pm}$.

To connect to a general definition of the SE in an arbitrary medium, we seek to obtain the Green's function defined through

$$
\begin{aligned}
\boldsymbol{\nabla} & \times \boldsymbol{\nabla} \times \mathbf{G}\left(\mathbf{r}, \mathbf{r}_{0}, \omega\right)-\frac{\omega^{2}}{c^{2}} \epsilon(\mathbf{r}, \omega) \mathbf{G}\left(\mathbf{r}, \mathbf{r}_{0}, \omega\right) \\
& =\frac{\omega^{2}}{c^{2}} \mathbf{1} \delta\left(\mathbf{r}-\mathbf{r}_{0}\right),
\end{aligned}
$$

with corresponding radiation conditions, and $\mathbf{1}$ is the unit tensor. The normalized QNMs can be used to define the Green's function for locations near (or within) the scattering geometry through $[61,87]$

$$
\mathbf{G}\left(\mathbf{r}, \mathbf{r}_{0}, \omega\right)=\sum_{\mu} A_{\mu}(\omega) \tilde{\mathbf{f}}_{\mu}(\mathbf{r}) \tilde{\mathbf{f}}_{\mu}\left(\mathbf{r}_{0}\right),
$$

with $A_{\mu}(\omega)=\omega /\left[2\left(\tilde{\omega}_{\mu}-\omega\right)\right]$. Expanding the Green's function with the QNMs can easily be used to compute the SE rate and the Purcell factor. We stress again that the medium must meet the condition for linear amplifying media, which coincides with a causal Green's function in the sense of linear response theory (Kramers-Kronig relations). Here, the Green's function must be analytic in the upper half complex plane to fulfill the Kramers-Kronig relations, and this can be rigorously justified by using a QNM approach. Indeed, without such an approach, it is not 
known whether the model represents a physically meaningful solution for Maxwell's equations.

Considering a dipole emitter $\mathbf{d}=d_{0} \mathbf{n}_{d}$ at location $\mathbf{r}_{d}$, the classical SE rate is [67]

$$
\Gamma\left(\mathbf{r}_{d}, \omega\right)=\frac{2}{\hbar \epsilon_{0}} \mathbf{d} \cdot \operatorname{Im}\left\{\mathbf{G}\left(\mathbf{r}_{d}, \mathbf{r}_{d}, \omega\right)\right\} \cdot \mathbf{d},
$$

and the generalized Purcell factor reads $[67,88]$

$$
F_{P}\left(\mathbf{r}_{d}, \omega\right)=1+\frac{\Gamma\left(\mathbf{r}_{d}, \omega\right)}{\Gamma_{0}\left(\mathbf{r}_{d}, \omega\right)},
$$

where $\Gamma_{0}\left(\mathbf{r}_{d}, \omega\right)=2 \mathbf{d} \cdot \operatorname{Im}\left\{\mathbf{G}_{B}\left(\mathbf{r}_{d}, \mathbf{r}_{d}, \omega\right)\right\} \cdot \mathbf{d} /\left(\hbar \epsilon_{0}\right)$, and $\mathbf{G}_{B}$ is the Green's function for a homogeneous medium (known analytically). For a 2D TM dipole, $\operatorname{Im}\left\{\mathbf{G}_{B}\left(\mathbf{r}_{d}, \mathbf{r}_{d}, \omega\right)\right\}=\omega^{2} / 4 c^{2}$. The factor of 1 appears naturally for dipole positions outside the resonator [89].

For an arbitrary photonic cavity medium, the QNMs for both the bare resonators (i.e., without coupling) and also for the coupled system can be obtained from an efficient dipole-scattering approach in complex frequency space [68] described in more detail in Appendix A. The total Green's function can also be obtained numerically from the full dipole response (i.e., without any modal approximations), which we carry out in COMSOL to check the accuracy of the QNM expansion form. Although the hybrid QNMs can be obtained numerically as well, it is far more insightful to develop a coupled-mode formalism to describe the coupling geometry.

\section{COUPLED-MODE THEORY WITH AN INTUITIVE GREEN'S FUNCTION EXPANSION}

\section{A. Wave equation and normal modes}

Before developing a QNMCMT using an intuitive Green's function approach, here we first present a NM approach and also connect to the common literature for describing when EPs can occur for coupled loss-gain cavity modes.

To simplify the equations and terminology, we introduce shorthand notation, and define the wave equation

$$
\mathcal{L}|\mathbf{E}\rangle=\omega^{2} \hat{\epsilon}_{t}|\mathbf{E}\rangle,
$$

where the fields are assumed to have a harmonic frequency dependence $e^{-i \omega t}, \mathcal{L}=c^{2} \boldsymbol{\nabla} \times \boldsymbol{\nabla} \times$, and $\epsilon_{t}(\mathbf{r})$ is the total dielectric constant that we assume is nondispersive. The operator $\hat{\epsilon}_{t}$ is defined as $\left\langle\mathbf{r}\left|\hat{\epsilon}_{t}\right| \mathbf{r}^{\prime}\right\rangle=\epsilon_{t}(\mathbf{r}) \delta\left(\mathbf{r}-\mathbf{r}^{\prime}\right)$, and the electric field is given by a projection onto space $\langle\mathbf{r} \mid \mathbf{E}\rangle=\mathbf{E}(\mathbf{r}, \omega)$.

To construct a Green's function solution, we consider a situation where we start with cavity 1 , and then add cavity 2. The dielectric constant defining cavity 1 is $\hat{\epsilon}_{1}=\hat{\epsilon}_{B}+\hat{V}_{1}$, so we can also write the wave equation as

$$
\left(\mathcal{L}-\omega^{2} \hat{\epsilon}_{1}\right)|\mathbf{E}\rangle=\omega^{2} \hat{V}_{2}|\mathbf{E}\rangle,
$$

where $\hat{V}_{2}$ defines the dielectric constant change after adding in cavity 2 , and we define $\epsilon_{B}=n_{B}^{2}$ as the entire background without either cavity. Naturally, we can also start from cavity 2 and add in cavity 1, and the end Green's function that includes both cavities must be the same.

\section{B. Coupled-mode theory and lossless exceptional points using normal modes}

Although the general CMT and Green's function derivations for coupled modes are generally well known, to make the QNM approach easier to understand and to better highlight the differences with a QNM approach, we first start with a NM approach. This also allows us to connect to the common theories for deriving an EP for coupledresonator modes.

Exploiting the fact that $\mathcal{L}$ is a linear self-adjoint operator over space, the homogeneous part of Eq. (7) defines an orthogonal set of eigenstates on a single cavity. It follows that

$$
\mathcal{L}\left|\mathbf{f}_{k}\right\rangle=\omega_{k}^{2} \hat{\epsilon}_{1}\left|\mathbf{f}_{k}\right\rangle,
$$

where $\omega_{k}$ are the eigenfrequencies of the eigenstates $\mathbf{f}_{k}$. These states are also complete and orthogonal [90], so

$$
\begin{aligned}
\sum_{k} \hat{\epsilon}_{1}\left|\mathbf{f}_{k}\right\rangle\left\langle\mathbf{f}_{k}\right| & =\mathbf{1}, \\
\left\langle\mathbf{f}_{i}\left|\hat{\epsilon}_{1}\right| \mathbf{f}_{j}\right\rangle & =\delta_{i j},
\end{aligned}
$$

and the sum includes all modes, physical and unphysical. Note the mode sum here is over all modes attached to the medium and resonator associated with $\epsilon_{1}$. A similar relation holds for the modes of resonator 2 .

Next, we can formulate a scattering problem for the field $|\mathbf{E}\rangle$ of the coupled resonator system, so that

$$
|\mathbf{E}\rangle=\left|\mathbf{E}^{0}\right\rangle+\hat{\mathbf{G}} \hat{V}_{2}\left|\mathbf{E}^{0}\right\rangle,
$$

where $\left|\mathbf{E}^{0}\right\rangle$ is the scattered field with resonator 1 only, and $\mathbf{G}\left(\mathbf{r}, \mathbf{r}^{\prime}\right)=\left\langle\mathbf{r}|\hat{\mathbf{G}}| \mathbf{r}^{\prime}\right\rangle$ is the total Green's function of the system (including both cavities) and is defined from

$$
\left(\mathcal{L}-\omega^{2} \hat{\epsilon}_{1}-\omega^{2} \hat{V}_{2}\right) \hat{\mathbf{G}}=\left(\mathcal{L}-\omega^{2} \hat{\epsilon}_{t}\right) \hat{\mathbf{G}}=\omega^{2} \mathbf{1} .
$$

For a hybrid system constructed from the coupled resonators of interest, we can expand $\hat{\mathbf{G}}$ in terms of a restricted set of carefully chosen basis states, which will be the dominant modes of the individual cavity systems [90]. Thus, one obtains the (NM) Green's function expansion

$$
\hat{\mathbf{G}}=\sum_{\alpha, \beta} B_{\alpha, \beta}\left|\mathbf{f}_{\alpha}\right\rangle\left\langle\mathbf{f}_{\beta}\right|,
$$

where both sums extend over all states of interest. If we obtain a solution for $B_{\alpha, \beta}$, then the scattering problem is 
solved as we have the total Green's function, including the new poles of the coupled-resonator system.

In the absence of cavity 2, we define the cavity mode of the bare cavity 1 (i.e., no cavity mode 2 yet), from

$$
\mathcal{L}\left|\mathbf{f}_{1}\right\rangle=\omega_{1}^{2} \hat{\epsilon}_{1}\left|\mathbf{f}_{1}\right\rangle,
$$

with the normalization $\left\langle\mathbf{f}_{1}\left|\hat{\epsilon}_{1}\right| \mathbf{f}_{1}\right\rangle=1$. For simplicity, we assume the cavity supports a single dominant mode in the frequency region of interest, but this can easily be generalized to allow for $N$ modes per cavity. Similarly, we can define the solution of cavity 2 from

$$
\mathcal{L}\left|\mathbf{f}_{2}\right\rangle=\omega_{2}^{2} \hat{\epsilon}_{2}\left|\mathbf{f}_{2}\right\rangle,
$$

with $\left\langle\mathbf{f}_{2}\left|\hat{\epsilon}_{2}\right| \mathbf{f}_{2}\right\rangle=1$.

Subsequently, we substitute the mode expansions into the main Green's function Eq. (11) to obtain

$$
\begin{aligned}
& \sum_{\alpha, \beta}\left[\omega_{\alpha}^{2}\left\langle\mathbf{f}_{i}\left|\hat{\epsilon}_{\alpha}\right| \mathbf{f}_{\alpha}\right\rangle-\omega^{2}\left\langle\mathbf{f}_{i}\left|\hat{\epsilon}_{t}\right| \mathbf{f}_{\alpha}\right\rangle\right] B_{\alpha, \beta}\left\langle\mathbf{f}_{\beta}\left|\hat{\epsilon}_{t}\right| \mathbf{f}_{j}\right\rangle \\
& =\omega^{2}\left\langle\mathbf{f}_{i}\left|\hat{\epsilon}_{t}\right| \mathbf{f}_{j}\right\rangle
\end{aligned}
$$

where $i, j$ refer to any basis states, and $\hat{\epsilon}_{\alpha}$ can refer to $\hat{\epsilon}_{1}=\hat{\epsilon}_{t}-\hat{V}_{2}$ or $\hat{\epsilon}_{2}=\hat{\epsilon}_{t}-\hat{V}_{1}$. Equation (15) defines a matrix equation whose poles correspond to the new eigenfrequencies of the composite system. To proceed, we exploit the fact that the modes are only weakly coupled to each other, and so

$$
\left\langle\mathbf{f}_{\alpha}\left|\hat{\epsilon}_{t}\right| \mathbf{f}_{\beta}\right\rangle=\delta_{\alpha \beta},
$$

which can typically be easily checked numerically. Note this approximation is not needed in general, but we have numerically checked that nondiagonal contributions are negligible, as is also confirmed with full numerical dipole solutions. Otherwise, it is also easy to include these terms, which just involves solving a more complex matrix, whose solution can still be obtain explicitly.

The matrix defined from Eq. (15), namely, $M B T=T$, has the solution $B_{\alpha, \beta}=\left[M^{-1}\right]_{\alpha, \beta}$, with elements

$$
\begin{aligned}
M_{\alpha, \alpha} & =\frac{1}{\omega^{2}}\left(\omega_{\alpha}^{2}-\omega^{2}\right), \\
M_{\alpha, \beta \neq \alpha} & =\frac{1}{\omega^{2}}\left(-\omega_{\beta}^{2}\left\langle\mathbf{f}_{\alpha}\left|\hat{V}_{\alpha}\right| \mathbf{f}_{\beta}\right\rangle\right) .
\end{aligned}
$$

Thus, the matrix $M$ is

$$
M=\frac{1}{\omega^{2}}\left(\begin{array}{cc}
\omega_{1}^{2}-\omega^{2} & -\omega_{2}^{2}\left\langle\mathbf{f}_{1}\left|\hat{V}_{1}\right| \mathbf{f}_{2}\right\rangle \\
-\omega_{1}^{2}\left\langle\mathbf{f}_{2}\left|\hat{V}_{2}\right| \mathbf{f}_{1}\right\rangle & \omega_{2}^{2}-\omega^{2}
\end{array}\right),
$$

and we define the intermode coupling rates

$$
\kappa_{\alpha \beta}=\frac{\omega_{\beta}}{2}\left\langle\mathbf{f}_{\alpha}\left|\hat{V}_{\alpha}\right| \mathbf{f}_{\beta}\right\rangle,
$$

for $\alpha, \beta=1,2$, and $\alpha \neq \beta$, so that

$$
M=\frac{1}{\omega^{2}}\left(\begin{array}{cc}
\omega_{1}^{2}-\omega^{2} & -2 \omega_{2} \kappa_{12} \\
-2 \omega_{1} \kappa_{21} & \omega_{2}^{2}-\omega^{2}
\end{array}\right) .
$$

Matrix inversion can be solved without approximations; however, it is appropriate to obtain an easier form within a rotating-wave approximation. Using $\omega_{\alpha}^{2}-\omega^{2} \approx$ $\left(\omega_{\alpha}-\omega\right) 2 \omega_{\alpha} \approx\left(\omega_{\alpha}-\omega\right) 2 \omega$, then

$$
M=\frac{2}{\omega}\left(\begin{array}{cc}
\omega_{1}-\omega & -\kappa_{12} \\
-\kappa_{21} & \omega_{2}-\omega
\end{array}\right),
$$

and we obtain an explicit solution for the Green's function expansion coefficients

$$
B_{\alpha, \beta}=\frac{\omega / 2}{\left(\omega-\omega_{+}\right)\left(\omega-\omega_{-}\right)}\left(\begin{array}{cc}
\omega_{2}-\omega & \kappa_{12} \\
\kappa_{21} & \omega_{1}-\omega
\end{array}\right),
$$

where the pole frequencies are

$$
\omega_{ \pm}=\frac{\omega_{1}+\omega_{2}}{2} \pm \frac{\sqrt{4 \kappa_{12} \kappa_{21}+\left(\omega_{1}-\omega_{2}\right)^{2}}}{2} .
$$

Finally, we note that for closed-cavity systems, unitarity of a Hermitian system also requires that $\kappa_{12}=\kappa_{21}^{*}$, and the pole frequencies are simply

$$
\omega_{ \pm}=\frac{\omega_{1}+\omega_{2}}{2} \pm \frac{\sqrt{4\left|\kappa_{12}\right|^{2}+\left(\omega_{1}-\omega_{2}\right)^{2}}}{2} .
$$

This concludes the derivation of the NM Green's function with weakly coupled cavities. With regard to EPs, if we now consider the case with two cavity systems, one with loss $-\gamma_{0}$ $\left(\omega_{1}=\omega_{0}-i \gamma_{0}\right)$ and one with a loss-compensating gain $+\gamma_{0}$ $\left(\omega_{2}=\omega_{0}+i \gamma_{0}\right)$, then one might be tempted to predict a situation where $\omega_{ \pm} \rightarrow \omega_{0}$, if $\left|\kappa_{12}\right|= \pm \gamma_{0}$. The problem with this argument is that the original cavity modes here do not satisfy a Hermitian eigenvalue problem (assuming they are open and/or contain some loss), and thus, the above coupledmode Green's function solutions are not valid. Strictly, they are only valid for real eigenfrequency cavity modes. For very high- $Q$ cavities, however, the theory may be approximately correct, but the definition of a true EP still becomes questionable. Moreover, even for high- $Q$ modes, the theory can completely fail, as we will show later.

\section{Coupled-mode theory and lossy exceptional points using quasinormal modes}

Since we are interested in open cavities with loss and gain, we now adopt a more rigorous and appropriate resonator approach using QNMs. One form of the QNM normalization can be defined from 


$$
\left\langle\left\langle\tilde{\mathbf{f}}_{1}\left|\hat{\epsilon}_{1}\right| \tilde{\mathbf{f}}_{1}\right\rangle\right\rangle \rightarrow \int d \mathbf{r} \epsilon_{1}(\mathbf{r}) \tilde{\mathbf{f}}_{1}(\mathbf{r}) \tilde{\mathbf{f}}_{1}(\mathbf{r})=1
$$

where some coordinate transform is applied to regularize the outgoing surface fields, e.g., through perfectly matched layers [66], but such terms are not needed in the region for CMT overlap integrals as we discuss below. One can also define this normalization in terms of electric and magnetic QNMs. Alternative QNM normalizations are discussed in Appendix A, including how to normalize with dispersive materials.

These fields are now solutions to the eigenvalue problem with complex frequencies, and virtually all of the previous equations apply, with some simple replacements:

(i) The eigenfrequencies become complex and formally discrete (although the NMs are also assumed to be discrete for resonator problems, formally they yield continuous eigenfrequencies):

$$
\omega_{k} \rightarrow \tilde{\omega}_{\mu},
$$

with $\tilde{\omega}_{\mu}=\omega_{\mu}-i \gamma_{\mu}\left(\gamma_{\mu}>0\left[\gamma_{\mu}<0\right]\right.$ for a lossy [gain] QNM).

(ii) The completeness relation (e.g., for resonator 1) becomes

$$
\begin{aligned}
& \frac{1}{2} \sum_{\mu= \pm 1,2, \ldots} \hat{\epsilon}_{1}\left|\tilde{\mathbf{f}}_{\mu}\right\rangle\left\langle\tilde{\mathbf{f}}_{\mu}^{*}\right|=\mathbf{1}, \\
& \left\langle\left\langle\tilde{\mathbf{f}}_{\mu}\left|\hat{\epsilon}_{1}\right| \tilde{\mathbf{f}}_{\eta}\right\rangle\right\rangle=\delta_{\mu \eta},
\end{aligned}
$$

which is assumed to be valid for spatial regions near or inside the scattering geometry. We note again that a similar relation holds for the modes of resonator 2, with a different set of QNMs.

(iii) The QNM Green's function expansion is

$$
\hat{\mathbf{G}}=\sum_{\alpha, \beta} \tilde{B}_{\alpha, \beta}\left|\tilde{\mathbf{f}}_{\alpha}\right\rangle\left\langle\tilde{\mathbf{f}}_{\beta}^{*}\right| .
$$

Thus, for example, the previous NM matrix equation (15) using QNMs now becomes

$$
\begin{aligned}
& \sum_{\alpha, \beta}\left[\tilde{\omega}_{\alpha}^{2}\left\langle\left\langle\tilde{\mathbf{f}}_{i}\left|\hat{\epsilon}_{\alpha}\right| \tilde{\mathbf{f}}_{\alpha}\right\rangle\right\rangle-\omega^{2}\left\langle\left\langle\tilde{\mathbf{f}}_{i}\left|\hat{\epsilon}_{t}\right| \tilde{\mathbf{f}}_{\alpha}\right\rangle\right\rangle\right] \tilde{B}_{\alpha, \beta}\left\langle\left\langle\tilde{\mathbf{f}}_{\beta}\left|\hat{\epsilon}_{t}\right| \tilde{\mathbf{f}}_{j}\right\rangle\right\rangle \\
& \quad=\omega^{2}\left\langle\left\langle\tilde{\mathbf{f}}_{i}\left|\hat{\epsilon}_{t}\right| \tilde{\mathbf{f}}_{j}\right\rangle\right\rangle .
\end{aligned}
$$

(iv) For regions sufficiently far outside the scattering geometry specifically, when $\exp \left(\gamma_{\alpha} s\right)$ becomes appreciable, one can use regularized QNMs (nondivergent), such that

$$
\hat{\mathbf{G}}=\sum_{\alpha, \beta} \tilde{B}_{\alpha, \beta}\left|\tilde{\mathbf{F}}_{\alpha}\right\rangle\left\langle\tilde{\mathbf{F}}_{\beta}^{*}\right|,
$$

where $\left|\tilde{\mathbf{F}}_{\alpha}\right\rangle$ is obtained from a Dyson solution using the original QNM [87] or using near-field to far-field transformations [91]. For high- $Q$ cavities, however, using the QNMs in the perturbative cavity region is in practice extremely accurate, as we also confirm later. Thus, in this work, we can safely use the QNMs for our overlap integral calculations.

Although the QNMs can be used here as an approximation for the overlap integrals, note that regularization is required in general. Also note that regularization is also required for solving the input-output relations with quantized QNMs [75-77,91,92], and similar potential problems also exist for QNM perturbation theories. For example, in the case of nondispersive materials [60,93], one has $\Delta \tilde{\omega}_{1}=-\tilde{\omega}_{1}\left\langle\left\langle\tilde{\mathbf{f}}_{1}|\Delta \epsilon| \tilde{\mathbf{f}}_{1}\right\rangle\right\rangle / 2$, which clearly has problems if the perturbation is added far away from the cavity region. In contrast, a regularized form can be first worked out using $\tilde{\mathbf{F}}_{1}(R, \omega)$ and then $\omega \approx \omega_{1}$. In this way, one obtains a convergent result, where it can also be shown that $\tilde{\mathbf{F}}_{1}\left(\mathbf{r}_{s}, \omega\right) \equiv \tilde{\mathbf{f}}_{1}\left(\mathbf{r}_{s}\right)$ at the surface between inside and outside the resonator.

With these replacements, we can use the same approach as before. Assuming again that the solution is first solved for cavity 1 , and then we add in cavity 2 , we derive

$$
\tilde{B}_{\alpha, \beta}=\frac{\omega / 2}{\left(\omega-\tilde{\omega}_{+}\right)\left(\omega-\tilde{\omega}_{-}\right)}\left(\begin{array}{cc}
\tilde{\omega}_{2}-\omega & \tilde{\kappa}_{12} \\
\tilde{\kappa}_{21} & \tilde{\omega}_{1}-\omega
\end{array}\right),
$$

where $(\alpha, \beta=1,2, \alpha \neq \beta)$

$$
\tilde{\kappa}_{\alpha \beta}=\frac{\tilde{\omega}_{\beta}}{2}\left\langle\left\langle\tilde{\mathbf{f}}_{\alpha}\left|\hat{V}_{\alpha}\right| \tilde{\mathbf{f}}_{\beta}\right\rangle\right\rangle,
$$

which notably now uses an unconjugated norm in the QNM overlap integrals. For the QNM formalism, note $\tilde{\kappa}_{12} \neq \tilde{\kappa}_{21}^{*}$ (see also Refs. [80,81]), in contrast to NM theory. In QNM theory, these off-diagonal terms are not the complex conjugates of each other, since the open cavity system does not obey Hermiticity. Indeed, using a conjugated norm with open cavities is simply ill-defined.

The full QNM Green's function solution [using Eqs. (31) and (28)] can be written as follows:

$$
\begin{aligned}
\hat{\mathbf{G}}= & \frac{\frac{\omega}{2}\left(\tilde{\omega}_{2}-\omega\right)\left|\tilde{\mathbf{f}}_{1}\right\rangle\left\langle\tilde{\mathbf{f}}_{1}^{*}\right|}{\left(\omega-\tilde{\omega}_{+}\right)\left(\omega-\tilde{\omega}_{-}\right)}+\frac{\frac{\omega}{2} \tilde{\kappa}_{12}\left|\tilde{\mathbf{f}}_{1}\right\rangle\left\langle\tilde{\mathbf{f}}_{2}^{*}\right|}{\left(\omega-\tilde{\omega}_{+}\right)\left(\omega-\tilde{\omega}_{-}\right)} \\
& +\frac{\frac{\omega}{2} \tilde{\kappa}_{21}\left|\tilde{\mathbf{f}}_{2}\right\rangle\left\langle\tilde{\mathbf{f}}_{1}^{*}\right|}{\left(\omega-\tilde{\omega}_{+}\right)\left(\omega-\tilde{\omega}_{-}\right)}+\frac{\frac{\omega}{2}\left(\tilde{\omega}_{1}-\omega\right)\left|\tilde{\mathbf{f}}_{2}\right\rangle\left\langle\tilde{\mathbf{f}}_{2}^{*}\right|}{\left(\omega-\tilde{\omega}_{+}\right)\left(\omega-\tilde{\omega}_{-}\right)},
\end{aligned}
$$

with the two new QNM pole frequencies for the composite cavity system,

$$
\tilde{\omega}_{ \pm}=\frac{\tilde{\omega}_{1}+\tilde{\omega}_{2}}{2} \pm \frac{\sqrt{4 \tilde{\kappa}_{12} \tilde{\kappa}_{21}+\left(\tilde{\omega}_{1}-\tilde{\omega}_{2}\right)^{2}}}{2} .
$$

In the limit of no coupling, $\tilde{\kappa}_{12}=\tilde{\kappa}_{21}=0$ and $\tilde{\omega}_{ \pm} \rightarrow \tilde{\omega}_{1,2}$, and we recover the original Green's function expansion for two separated resonators. 
Finally, we briefly discuss the modified condition for EPs. Equation (34) shows that the exceptional point occurs when

$$
\left.2 \sqrt{\tilde{\kappa}_{21} \tilde{\kappa}_{12}}\right|_{\mathrm{EP}} \equiv A+i B= \pm i\left(\tilde{\omega}_{1}-\tilde{\omega}_{2}\right),
$$

and in general one might obtain a lossy EP (at best), since the resonators are open, and the coupled system must also yield a finite loss for any linear amplifying medium [1]. The influence of dissipation on EPs and CMT is often argued heuristically. For example (defining $\tilde{\omega}_{1}=\omega_{1}-i \gamma_{1}$ and $\tilde{\omega}_{2}=\omega_{2}-i \gamma_{2}$ ), Ref. [32] discusses two limits where (i) the coupling is real, i.e., $B=0$, so the $\mathrm{EP}$ condition would become $A= \pm 2 \gamma_{0}$, with $\gamma_{1}=\gamma_{0}$ and $\gamma_{2}=-\gamma_{0}$ $\left(\omega_{1}=\omega_{2}=\omega_{0}\right)$, and (ii) where the coupling is purely imaginary, i.e., $A=0$; then the EPs' condition would become $B= \pm \Delta$, where $\omega_{1}=\omega_{0}+\Delta$ and $\omega_{2}=\omega_{0}$ $\left(\gamma_{1}=\gamma_{2}\right)$. In practice, it would be very difficult to reach exactly this regime [Eq. (35)], though one can likely come close and certainly find signatures of EP-like behavior.

Experimentally, in Refs. [38,39,94], the spectra below and above EPs are observed, as well as the associated unidirectional transmission, but an exact EP was not demonstrated. Note, however, that these systems involve waveguides, so the CMT theory should be modified to connect more closely to such works. The theory in Ref. [46] also finds nonideal EPs if one shows an enlargement of the EP region in the complex frequency plane.

For a high- $Q$ resonator, one can assume that an approximate EP may be obtained when $\operatorname{Re}\left(\tilde{\kappa}_{12}\right)= \pm \gamma_{0}$ (assuming $\tilde{\omega}_{1}=\omega_{0}-i \gamma_{0}$ and $\left.\tilde{\omega}_{2}=\omega_{0}+i \gamma_{0}\right)$, but in general, this condition is an approximate one for open resonators (and QNMs). Moreover, in the vicinity of an EP, the line shapes associated with the QNMs are far richer than with two coupled Lorentzian line shapes, as we demonstrate in more detail below, even for very high- $Q$ modes $\left(Q \approx 10^{5}\right)$.

\section{Hybrid quasinormal modes using the coupled-mode theory}

Next, we introduce a model for obtaining the hybridized QNMs, which can be obtained analytically in terms of the uncoupled QNMs; this considerably simplifies the numerical solutions and helps identify the underlying physics of how the hybridized modes are formed.

In terms of the hybridized eigenfrequencies $\tilde{\omega}_{ \pm}$defined in Eq. (34), we obtain

$$
\begin{aligned}
\left|\tilde{\mathbf{f}}^{ \pm}\right\rangle= & \frac{\tilde{\omega}_{ \pm}-\tilde{\omega}_{2}}{\sqrt{\left(\tilde{\omega}_{ \pm}-\tilde{\omega}_{2}\right)^{2}+\tilde{\kappa}_{21}^{2}}}\left|\tilde{\mathbf{f}}_{1}\right\rangle \\
& +\frac{-\tilde{\kappa}_{21}}{\sqrt{\left(\tilde{\omega}_{ \pm}-\tilde{\omega}_{2}\right)^{2}+\tilde{\kappa}_{21}^{2}}}\left|\tilde{\mathbf{f}}_{2}\right\rangle
\end{aligned}
$$

or

$$
\begin{aligned}
\left|\tilde{\mathbf{f}}^{ \pm}\right\rangle= & \frac{-\tilde{\kappa}_{12}}{\sqrt{\left(\tilde{\omega}_{ \pm}-\tilde{\omega}_{1}\right)^{2}+\tilde{\kappa}_{12}^{2}}}\left|\tilde{\mathbf{f}}_{1}\right\rangle \\
& +\frac{\tilde{\omega}_{ \pm}-\tilde{\omega}_{1}}{\sqrt{\left(\tilde{\omega}_{ \pm}-\tilde{\omega}_{1}\right)^{2}+\tilde{\kappa}_{12}^{2}}}\left|\tilde{\mathbf{f}}_{2}\right\rangle .
\end{aligned}
$$

Assuming $\tilde{\kappa}=\tilde{\kappa}_{12} \approx \tilde{\kappa}_{21}$, then

$$
\begin{aligned}
\left|\tilde{\mathbf{f}}^{ \pm}\right\rangle= & \frac{-\tilde{\kappa}}{\sqrt{\left(\tilde{\omega}_{ \pm}-\tilde{\omega}_{1}\right)^{2}+\tilde{\kappa}^{2}}}\left|\tilde{\mathbf{f}}_{1}\right\rangle \\
& +\frac{\tilde{\omega}_{ \pm}-\tilde{\omega}_{1}}{\sqrt{\left(\tilde{\omega}_{ \pm}-\tilde{\omega}_{1}\right)^{2}+\tilde{\kappa}^{2}}}\left|\tilde{\mathbf{f}}_{2}\right\rangle,
\end{aligned}
$$

which applies to all inspected examples. But we are using Eqs. (36) or (37) in Sec. V.

Subsequently, we also obtain the new Green's function

$$
\hat{\mathbf{G}}=\frac{\omega\left|\tilde{\mathbf{f}}^{+}\right\rangle\left\langle\tilde{\mathbf{f}}^{+*}\right|}{2\left(\tilde{\omega}_{+}-\omega\right)}+\frac{\omega\left|\tilde{\mathbf{f}}^{-}\right\rangle\left\langle\tilde{\mathbf{f}}^{-*}\right|}{2\left(\tilde{\omega}_{-}-\omega\right)},
$$

which gives the same results as Eq. (33) (apart from at an $\mathrm{EP}$, which is discussed later, as the hybrid modes become self-orthogonal), but now in diagonalized form.

\section{GREEN'S FUNCTIONS AND PURCELL FACTORS AT THE EXCEPTIONAL POINT}

The SE rates (and generalized Purcell factors) can be significantly modified close to EPs [82-84,95-97], where a squared-Lorentzian contribution has been emphasized as well as signatures of linewidth narrowing. With higherorder EPs, these linewidths may be reduced further [83]; e.g., a cubic Lorentzian line shape has been predicted with third-order EPs [96].

Below, we focus on the more general second-order EPs and first briefly comment on previous theoretical predictions about the modified line shapes. In Ref. [84], the frequencydependent response of SE has the following form:

$$
\tilde{S}(\omega)=\frac{A}{\left(\omega-\omega_{k}\right)^{2}}-\frac{B}{\left(\omega-\omega_{k}\right)},
$$

which is a complex Lorentzian squared and a single Lorentzian, if very near the EP.

A similar regime was predicted and shown numerically in Ref. [83]. The main Green's function response was predicted to have the following form:

$$
G(\omega)=\frac{C}{\omega^{2}-\tilde{\omega}_{\mathrm{EP}}^{2}}+\frac{D}{\left(\omega^{2}-\tilde{\omega}_{\mathrm{EP}}^{2}\right)^{2}},
$$

where $C$ and $D$ are connected with the Jordan vectors. They also give an approximate coupled-mode theory expression for the Green's function expansion.

Next, we first show how our QNM Green's function is fully consistent with the above predictions and then show why one can find a much richer range of complex line 
shapes, which we also demonstrate explicitly in the numerical results section.

We define the EP resonance frequency from $\tilde{\omega}_{ \pm}=$ $\tilde{\omega}_{\mathrm{EP}}=\left(\tilde{\omega}_{1}+\tilde{\omega}_{2}\right) / 2$, which occurs when $\tilde{\Delta}_{12}^{2} \equiv\left(\tilde{\omega}_{1}-\tilde{\omega}_{2}\right)^{2}=$ $-4 \tilde{\kappa}_{12} \tilde{\kappa}_{21}$. Thus, we can write

$$
M=\frac{2}{\omega}\left(\begin{array}{cc}
\tilde{\omega}_{\mathrm{EP}}-\omega+\tilde{\omega}_{\mathrm{EP}}-\tilde{\omega}_{2} & -\tilde{\kappa}_{12} \\
-\tilde{\kappa}_{21} & \tilde{\omega}_{\mathrm{EP}}-\omega+\tilde{\omega}_{\mathrm{EP}}-\tilde{\omega}_{1}
\end{array}\right),
$$

which is identical to what we did already, but this form allows us to expand the Green's function near the EP resonances. For example, the $\hat{\mathbf{G}}_{1,1}$ term (expanded in terms of the bare mode from resonator 1) is

$$
\hat{\mathbf{G}}_{1,1}=\frac{\omega}{2}\left(\frac{\tilde{\omega}_{\mathrm{EP}}-\tilde{\omega}_{1}}{\left(\omega-\tilde{\omega}_{\mathrm{EP}}\right)^{2}}-\frac{1}{\omega-\tilde{\omega}_{\mathrm{EP}}}\right)\left|\tilde{\mathbf{f}}_{1}\right\rangle\left\langle\tilde{\mathbf{f}}_{1}^{*}\right|,
$$

where we clearly see the separation of a Lorentzian and a Lorentzian-squared contribution, in agreement with Refs. [82-84].

We stress that our spectral forms are not actually Lorentzian or Lorentzian squared because of the QNM phase. Indeed, given the appropriate QNM phase, these terms can contribute negatively, a feature that is already known with coupled QNMs yielding a Fano resonance [91,98-101]. It is also useful to compare with the response of the single-cavity solution, which is simply

$$
\hat{\mathbf{G}}^{\mathrm{cav} 1}=\left(\frac{\omega}{2\left(\tilde{\omega}_{1}-\omega\right)}\right)\left|\tilde{\mathbf{f}}_{1}\right\rangle\left\langle\tilde{\mathbf{f}}_{1}^{*}\right|,
$$

which has a single Lorentzian-like feature, again modified by the QNM phase. Thus, the Lorentzian-squared feature is caused by the EP coupling regime.

Since $\tilde{\omega}_{\mathrm{EP}}=\left(\tilde{\omega}_{1}+\tilde{\omega}_{2}\right) / 2$, we can also write the QNM Green's function solution to the coupled-resonator EP regime as

$$
\begin{aligned}
& \hat{\mathbf{G}}_{1,1}=\frac{\omega}{2}\left(\frac{-\tilde{\Delta}_{12} / 2}{\left(\tilde{\omega}_{\mathrm{EP}}-\omega\right)^{2}}+\frac{1}{\left(\tilde{\omega}_{\mathrm{EP}}-\omega\right)}\right)\left|\tilde{\mathbf{f}}_{1}\right\rangle\left\langle\tilde{\mathbf{f}}_{1}^{*}\right|, \\
& \hat{\mathbf{G}}_{1,2}=\frac{\omega}{2}\left(\frac{\tilde{\kappa}_{12}}{\left(\tilde{\omega}_{\mathrm{EP}}-\omega\right)^{2}}\right)\left|\tilde{\mathbf{f}}_{1}\right\rangle\left\langle\tilde{\mathbf{f}}_{2}^{*}\right|, \\
& \hat{\mathbf{G}}_{2,1}=\frac{\omega}{2}\left(\frac{\tilde{\kappa}_{21}}{\left(\tilde{\omega}_{\mathrm{EP}}-\omega\right)^{2}}\right)\left|\tilde{\mathbf{f}}_{2}\right\rangle\left\langle\tilde{\mathbf{f}}_{1}^{*}\right|, \\
& \hat{\mathbf{G}}_{2,2}=\frac{\omega}{2}\left(\frac{\tilde{\Delta}_{12} / 2}{2\left(\tilde{\omega}_{\mathrm{EP}}-\omega\right)^{2}}+\frac{1}{\left(\tilde{\omega}_{\mathrm{EP}}-\omega\right)}\right)\left|\tilde{\mathbf{f}}_{2}\right\rangle\left\langle\tilde{\mathbf{f}}_{2}^{*}\right|,
\end{aligned}
$$

with $\tilde{\Delta}_{12}=\tilde{\omega}_{1}-\tilde{\omega}_{2}$. Note that any divergences from the hybrid modes at the EP are avoided here, since we use an expansion in terms of the bare modes and coupling parameters from CMT.
Interestingly, we obtain this known (and highly unusual) form without having to perform any Jordan expansion around the EP pole $[83,84,95]$. Assuming the CMT is accurate (and we show below that it can be quantitatively accurate), this is clearly a more convenient form to work with. It is also important to note that the hybrid QNM modes, e.g., in Eq. (36), diverge at the EP, since the QNMs at exactly this point are ill-defined and self-orthogonal. In practice, however, this is not a restriction, as most solutions will deviate from precisely this point, where the two mode Green's function responses become identical and well defined; thus, one can use either the bare modes (nondiagonal form) or the hybrid mode solutions (diagonal form). This is a significant advantage that benefits from the constructed CMT.

In the next section, we highlight much more general forms of the spectral line shapes near the EP, which are fully verified by numerically exact solutions (within numerical precision). Our calculations also point out a fundamental problem with defining a SE rate in media with coupled loss and gain resonators, even when the total Green's function is analytic in the upper complex half plane [1].

\section{NUMERICAL RESULTS FOR COUPLED LOSS-GAIN MICRODISK RESONATORS}

We consider two coupled loss-gain microdisk resonators, both with a disk radius of $R=5 \mu \mathrm{m}$ (cf. Fig. 2). The refractive index of the lossy (gain) resonator is $n_{\text {loss }}=$ $2+10^{-5} i\left(n_{\text {gain }}=2-5 \times 10^{-6} i\right)$, unless stated otherwise. The background medium is free space with $n_{B}=1$. The gap distance between the resonators is $d_{\text {gap }}$ (around 1120-1200 nm). The dipole (out-of-plane line current, a point in 2D, shown as red dot in Fig. 2) is placed within the

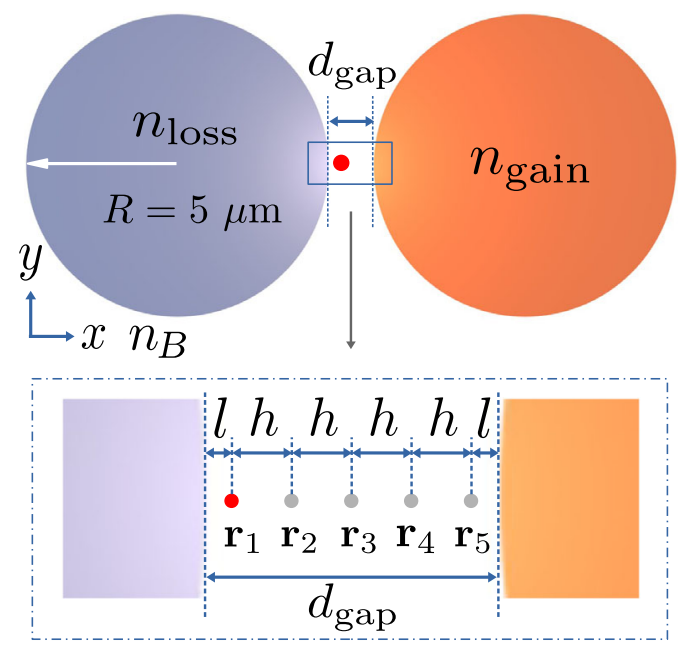

FIG. 2. Schematic diagram of the considering coupled loss and gain 2D microdisk resonator system. The radius of both resonators is $R=5 \mu \mathrm{m}$. The gap distance between the resonators is $d_{\text {gap }}$, and the dipole (emitter) is placed within the gap at several possible positions: $\mathbf{r}_{1}, \mathbf{r}_{2}, \mathbf{r}_{3}, \mathbf{r}_{4}$, and $\mathbf{r}_{5}$ (see text). The origin of the coordinate system is at the gap center. 
gap at several possible positions: $\mathbf{r}_{d}=\mathbf{r}_{1}, \mathbf{r}_{2}, \mathbf{r}_{3}, \mathbf{r}_{4}$, and $\mathbf{r}_{5}$, where $l=10 \mathrm{~nm}$ and $h=\left(d_{\text {gap }}-2 l\right) / 4\left(\mathbf{r}_{3}\right.$ is at gap center $)$.

\section{A. Quasinormal modes for single-loss and single-gain resonators}

Before investigating the coupled-resonator regime, we first show the QNMs for the single-loss or -gain resonators, which are used as input for the CMT in the next subsection.

A single 2D microdisk resonator supports WGMs $[102,103]$, which generally can be described by three mode numbers: radial mode number $q(=1,2,3 \ldots)$, azimuthal mode number $m$, and polarization $p$ (TM or TE). Note that for the same $q, m$, and $p$, there are two degenerate counterpropagating modes [61,104-106]: $E_{\mathrm{CW}}(\mathbf{r}, \phi)=E(\mathbf{r}) e^{(-i m \phi)}$ with clockwise $(\mathrm{CW})$ direction and $E_{\mathrm{CCW}}(\mathbf{r}, \phi)=E(\mathbf{r}) e^{(i m \phi)}$ with counterclockwise $(\mathrm{CCW})$ direction. For a TM mode, there is only the $z$ component for electric fields, which share the same eigenvalues. Linear combinations of these modes result in degenerate standing waves [104-106], such as a symmetric standing-wave mode $E_{s}=E_{\mathrm{CW}}+E_{\mathrm{CCW}} \propto$ $\cos (m \phi)$, and an antisymmetric standing-wave mode $E_{\mathrm{AS}}=$ $E_{\mathrm{CW}}-E_{\mathrm{CCW}} \propto \sin (m \phi)$.

In general, the mode with $q=1$ and $m \gg 1$ has a high $Q$ and strong field confinement. In this work, we focus on a TM mode $\left(H_{x}, H_{y}, E_{z}\right)$ with $q=1$ and $m=37$ (yielding a resonant wavelength near the telecommunication band, around $1487 \mathrm{~nm}$ ). To compute the normalized QNMs, we employ an efficient dipole-scattering approach to obtain the QNMs in complex frequency space [68], where an outof-plane line current (a point in 2D, $z$ polarized) is placed $10 \mathrm{~nm}$ away from the 2D microdisk (the dipole is at $\mathbf{r}_{d}=\mathbf{r}_{1}$ for the single-lossy cavity and at $\mathbf{r}_{d}=\mathbf{r}_{5}$ for the single-gain cavity). For more details, see Appendix A 1.

Conveniently, for our TM dipole location along the $x$ axis, we excite only one of the two degenerate standing-wave modes, as shown in Fig. 3(a) for the single-lossy cavity, which has the form $\cos (m \phi)$ if one considers $\phi=0$ at the positive $x$ axis. The orthogonal and degenerate QNM has the form $\sin (m \phi)$ constructed from an asymmetric linear combination of the clockwise and counterclockwise modes, though we need to consider only the symmetric QNM for our chosen dipole locations below. More details can be found in Appendix B. Also note that this working QNM dominates in the frequency region of interest below, because the two closest modes are $q=4, m=25$ and $q=3, m=29$, and the angular frequency spacing between them and the working mode are $\Delta \omega=$ $3.43 \times 10^{12} \mathrm{rad} / \mathrm{s}$ and $\Delta \omega=1.96 \times 10^{13} \mathrm{rad} / \mathrm{s}$, which are much larger than the FWHM (approximately $1.252 \times$ $10^{10} \mathrm{rad} / \mathrm{s}$ for the single-lossy cavity, and approximately $6.26 \times 10^{9} \mathrm{rad} / \mathrm{s}$ for the single-gain cavity). Also note, the free spectral range for modes with $q=1$ is also much larger than these FWHM values; e.g., the angular frequency spacing between $q=1, m=36$ (or $q=1, m=38$ ) with the working mode $(q=1, m=37)$ would be around $\Delta \omega=3.16 \times 10^{13} \mathrm{rad} / \mathrm{s}$. Thus, we can adopt a single
QNM approximation for the mode of interest for each resonator, which we also verify below by performing full dipole calculations with no mode approximations.

Numerically, the complex angular eigenfrequency for a single-lossy resonator is found to be $\tilde{\omega}_{L}=\omega_{L}-i \gamma_{L} \equiv$ $\omega_{0}-i \gamma_{0}=1.266666 \times 10^{15}-6.260269 \times 10^{9} i \mathrm{rad} / \mathrm{s}$, with a quality factor $Q \sim 10^{5}$; here, $\omega_{0}\left(\gamma_{0}\right)$ is the real part (opposite imaginary part) of the complex eigenfrequency. The corresponding QNM field distribution (real part $\operatorname{Re}\left[\tilde{f}_{z}^{L}\right]$ ), is shown in Fig. 3(a); the imaginary part is much smaller than the real part and thus is not shown.

To better understand the overlap integrals for use in CMT, the QNMs at the second resonator region are also shown (1155 nm away, i.e., $d_{\text {gap }}=1155 \mathrm{~nm}$ ), which is hardly seen on a linear scale because they are very small compared with fields close to the lossy resonator. This also indicates that the lossy QNMs show no sign of a spatial divergence in this region, and thus they can be accurately used for input to CMT (without regularization), which is a consequence of the high $Q$.

Similarly, the complex angular eigenfrequency for the single-gain resonator is found at $\tilde{\omega}_{G}=\omega_{G}-i \gamma_{G} \sim$ $\omega_{0}+i 0.5 \gamma_{0}$. The corresponding QNM field distribution (real part $\operatorname{Re}\left[\tilde{f}_{z}^{G}\right]$ ) is shown in Fig. 3(b). In addition, the Purcell factors at the dipole location (10 nm away from the loss and gain resonator) using a single QNM contribution [Eq. (5)] agree quantitatively well with full dipole results (see Appendix D). We also highlight that the Purcell factors (as defined in a semiclassical model) are net negative for the single-gain cavity only (since the field is being amplified rather than dissipated), a regime that is also shown below for the coupled-resonator system.

\section{B. Hybrid quasinormal modes for coupled loss-gain resonators}

We next study the hybrid QNMs formed from the two coupled microdisk resonators, using the QNM CMT and also using full numerical solutions to confirm the accuracy of our semianalytical results.

Using only the QNMs from single-lossy or -gain cavities as input, the properties for the coupled modes are obtained analytically. First, the new angular eigenfrequencies $\tilde{\omega}_{ \pm}$are computed from Eq. (34), where the coupling coefficients $\tilde{\kappa}_{12}$ and $\tilde{\kappa}_{21}$ [both complex, Eq. (32)] are related to the overlap QNM integrals for different gap distances $d_{\text {gap }}$. Note the input variables $\tilde{\omega}_{1 / 2}$ and $\tilde{\mathbf{f}}_{1 / 2}$ in theory parts are changed to $\tilde{\omega}_{L / G}$ and $\tilde{\mathbf{f}}^{L / G}$ in the numerical parts accordingly. As shown in Figs. 3(e) and 3(f), the analytical eigenfrequencies [Eq. (34)] versus full numerical solution in COMSOL (eigenfrequency solver) show excellent agreement, and at all gap separations. This quantitative level of agreement is also obtained for the QNMs and the QNM Green's functions as we show in more detail below.

The complex coupling coefficients for $d_{\text {gap }}=1155 \mathrm{~nm}$ $\left(d_{\text {gap }}=1160 \mathrm{~nm}\right)$ are $\tilde{\kappa}_{12} / \gamma_{0}=-0.7614-5.745 \times 10^{-6} i$ 
(a) $\operatorname{Re}\left[\tilde{f}_{z}^{L}\right] \quad \times 10^{5}\left(\mathrm{~m}^{-1}\right)$

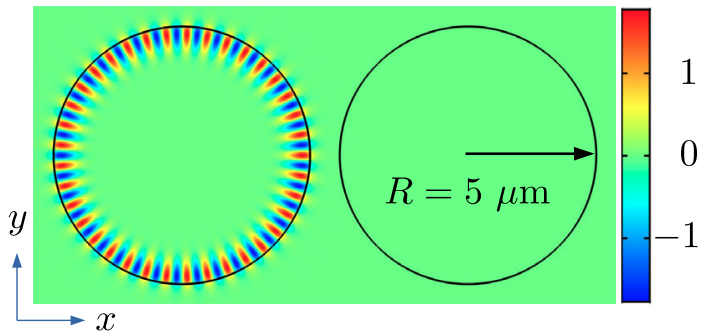

(c)

$$
\operatorname{Re}\left[\tilde{f}_{z}^{+}\right]
$$

$\times 10^{5}\left(\mathrm{~m}^{-1}\right)$
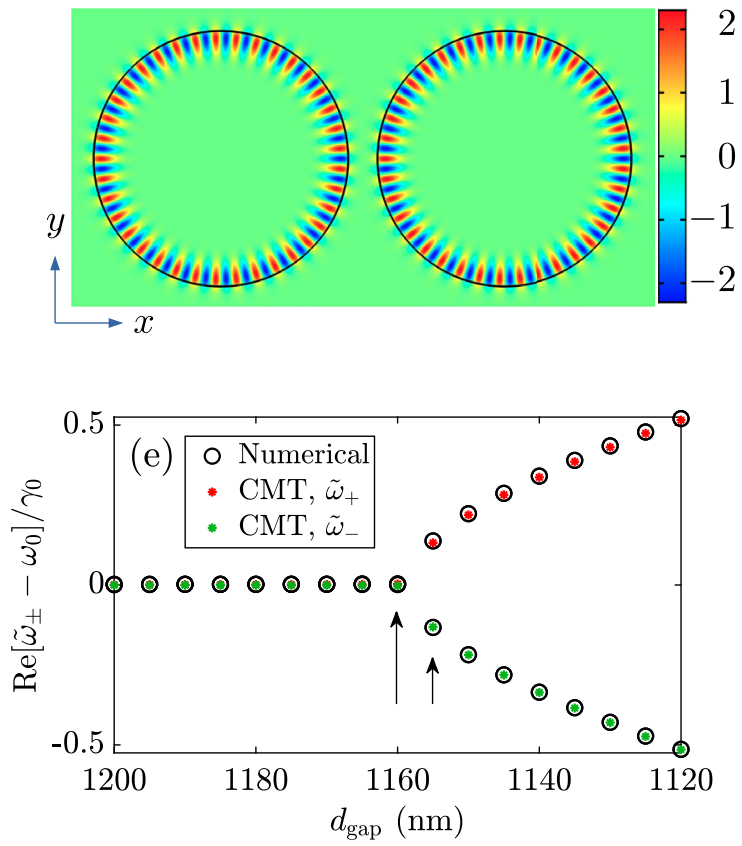

(b) $\operatorname{Re}\left[\tilde{f}_{z}^{G}\right] \quad \times 10^{5}\left(\mathrm{~m}^{-1}\right)$

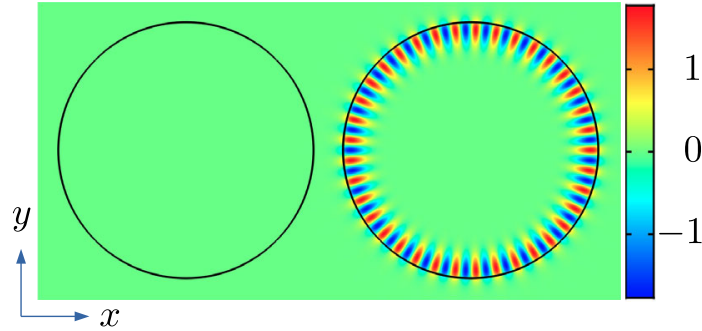

(d)

$$
\operatorname{Re}\left[\tilde{f}_{z}^{-}\right] \quad \times 10^{5}\left(\mathrm{~m}^{-1}\right)
$$
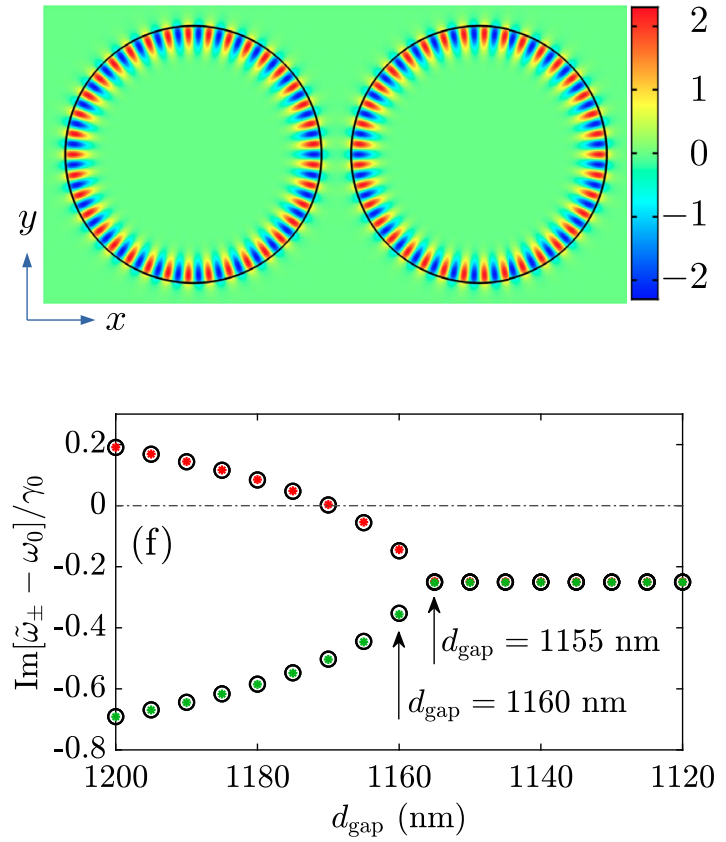

FIG. 3. Spatial profile of the QNMs for the following cavity configurations: (a) lossy resonator only $\left[\operatorname{Re}\left[\tilde{f}_{z}^{L}\right]\right.$ (the cavity on the right is shown for clarity and for use in the QNM CMT, but of course, it is not there for the single-resonator calculations), Eq. (A5)], (b) gain resonator only $\left[\operatorname{Re}\left[\tilde{f}_{z}^{G}\right]\right.$, Eq. (A5)], and (c),(d) hybrid modes with coupling $\left[\operatorname{Re}\left[\tilde{f}_{z}^{ \pm}\right]\right.$, Eq. (36)] for a separation distance $d_{\text {gap }}=1155 \mathrm{~nm}$; this regime is close to the EP as shown in (e) and (f). (e),(f) Complex QNM eigenfrequencies of the coupled microdisk resonators from a direct numerical eigenfrequency solver in COMSOL (numerical, black circles) and the analytical QNM CMT [Eq. (34), red stars for $\tilde{\omega}_{+}$, green stars for $\left.\tilde{\omega}_{-}\right]$, as a function of the gap separation $d_{\text {gap }}$. Note that using the eigenfrequency solver, there are two bare degenerate modes per resonator and four new eigenfrequencies for coupled resonators (four black circles for each gap distance), but with two pairs of degenerate modes. For the dipole QNM technique, only one of the degenerate standing-wave QNMs are obtained and used for single resonator (see text and Appendix A); thus, the analytical CMT produces two new eigenfrequencies in the presence of finite coupling (one red star and one green star for each gap distance). The arrows show two solutions near an EP. Note that $\omega_{0}$ and $\gamma_{0}$ are for the uncoupled single-lossy resonator with $n_{\text {loss }}=2+10^{-5} i\left(\tilde{\omega}_{L}=\omega_{L}-i \gamma_{L}=\omega_{0}-i \gamma_{0}\right)$, while for the uncoupled single-gain cavity with $n_{\text {gain }}=2-5 \times 10^{-6} i$, the resonance is around $\tilde{\omega}_{G} \approx \omega_{0}+i 0.5 \gamma_{0}$.

$\left(-0.7425-5.375 \times 10^{-6} i\right) \quad$ and $\quad \tilde{\kappa}_{21} / \gamma_{0}=-0.7614+$ $4.153 \times 10^{-5} i\left(-0.7425+4.087 \times 10^{-6} i\right)$, where the small imaginary part is mainly due to the high quality factor of the bare resonator, and one can find they do not satisfy $\tilde{\kappa}_{12}=\tilde{\kappa}_{21}^{*}$ in general, as mentioned in Sec. III C. This is also true for balanced loss-gain systems, and these inconspicuous imaginary parts would affect the condition for finding a perfect EP, e.g., as in Ref. [46].

Note, with a direct QNM eigenfrequency solver, there would be four new (dominant) eigenfrequencies for the coupled-resonator system, because there are two degenerate standing modes per resonator (see Appendix B); thus, there are four black circles for each gap distance in Figs. 3(e) and 3(f) but with two pairs of degenerate modes. In contrast, with the QNM dipole technique (see Appendix A), only one of the degenerate standing modes is used. Hence, when combining with the analytical CMT approach, there are only two new eigenfrequencies for the coupled-resonator system-labeled by the red star and the green star for each gap distance in Figs. 3(e) and 3(f). 
Equation (36) gives the two new coupled QNMs $\tilde{\mathbf{f}}^{ \pm}$ (hybrid QNMs) corresponding to the two new eigenfrequencies $\tilde{\omega}_{ \pm}$, where the input fields $\tilde{\mathbf{f}}_{1}$ and $\tilde{\mathbf{f}}_{2}$ are now $\tilde{\mathbf{f}}^{L}$ and $\tilde{\mathbf{f}}^{G}$. The spatial profile of the coupled QNMs (real part $\operatorname{Re}\left[\tilde{f}_{z}^{ \pm}\right]$) for $d_{\text {gap }}=1155 \mathrm{~nm}$ are shown in Figs. 3(c) and 3(d), where the fields now extend over both resonators. Below, we study two example gap cases close to the lossy EP, namely, $d_{\text {gap }}=1155 \mathrm{~nm}$ and $d_{\text {gap }}=1160 \mathrm{~nm}$ indicated by the arrows in Figs. 3(e) and 3(f).

\section{Non-Lorentzian Purcell factors close to a lossy exceptional point: Quasinormal mode Green's function solution versus full dipole simulations}

Next, we focus on the Purcell factors close to the lossy EPs in the coupled loss-gain resonators. Once the coupled QNMs [Eq. (36)] are obtained from CMT (the input fields $\tilde{\mathbf{f}}_{1}$ and $\tilde{\mathbf{f}}_{2}$ are now $\tilde{\mathbf{f}}^{L}$ and $\tilde{\mathbf{f}}^{G}$ ), the generalized Purcell factors are obtained analytically from Eq. (5) using the QNM Green's function [Eq. (39)]. As shown in Fig. 2, we consider five potential dipole positions along the $x$ axis and study the Purcell factors as a function of the frequency in each case.

For a dipole at the position $\mathbf{r}_{d}=\mathbf{r}_{1}(10 \mathrm{~nm}$ away from the lossy cavity), the Purcell factors are shown in Fig. 4(a) with $d_{\text {gap }}=1155 \mathrm{~nm}$, where negative Purcell factors (black solid curve) are found in a wide range of frequencies. The black solid line shows the analytical QNM Green's function result using only the bare resonator parameters as input, and the red circles are presenting full dipole solutions, where they are showing quantitatively good agreement over all frequencies. We stress there are no fitting parameters in the QNM solutions. Moreover, from our theory, the contribution from the hybrid QNMs $\tilde{\mathbf{f}}^{+}$and $\tilde{\mathbf{f}}^{-}$can be shown separately, as indicated by the green and blue solid curves [Eq. (39), the first and second term]. As a reference, we also show the Purcell factors for a single-lossy cavity (orange dashed curve), which is net positive and multiplied by 8 for a better graphical comparison. The gain resonator clearly acts to suppress the broadening and enhance the overall Purcell factors.

To help explain these unusual line shapes, we can study the contributions to the QNM Green's functions from the phases for the two hybrid QNMs. Defining $\tilde{f}_{z}^{+}(\mathbf{r})=\left|\tilde{f}_{z}^{+}(\mathbf{r})\right| e^{i \phi^{+}(\mathbf{r})}$ and $\tilde{f}_{z}^{-}(\mathbf{r})=\left|\tilde{f}_{z}^{-}(\mathbf{r})\right| e^{i \phi^{-}(\mathbf{r})}$, the QNM Green's function can be expressed as

$$
\begin{aligned}
& G_{z z}\left(\mathbf{r}_{d}, \mathbf{r}_{d}, \omega\right) \\
& =A^{+}(\omega) \tilde{f}_{z}^{+}\left(\mathbf{r}_{d}\right) \tilde{f}_{z}^{+}\left(\mathbf{r}_{d}\right)+A^{-}(\omega) \tilde{f}_{z}^{-}\left(\mathbf{r}_{d}\right) \tilde{f}_{z}^{-}\left(\mathbf{r}_{d}\right) \\
& =A^{+}(\omega) e^{i 2 \phi^{+}\left(\mathbf{r}_{d}\right)}\left|\tilde{f}_{z}^{+}\left(\mathbf{r}_{d}\right)\right|^{2}+A^{-}(\omega) e^{i 2 \phi^{-}\left(\mathbf{r}_{d}\right)}\left|\tilde{f}_{z}^{-}\left(\mathbf{r}_{d}\right)\right|^{2},
\end{aligned}
$$
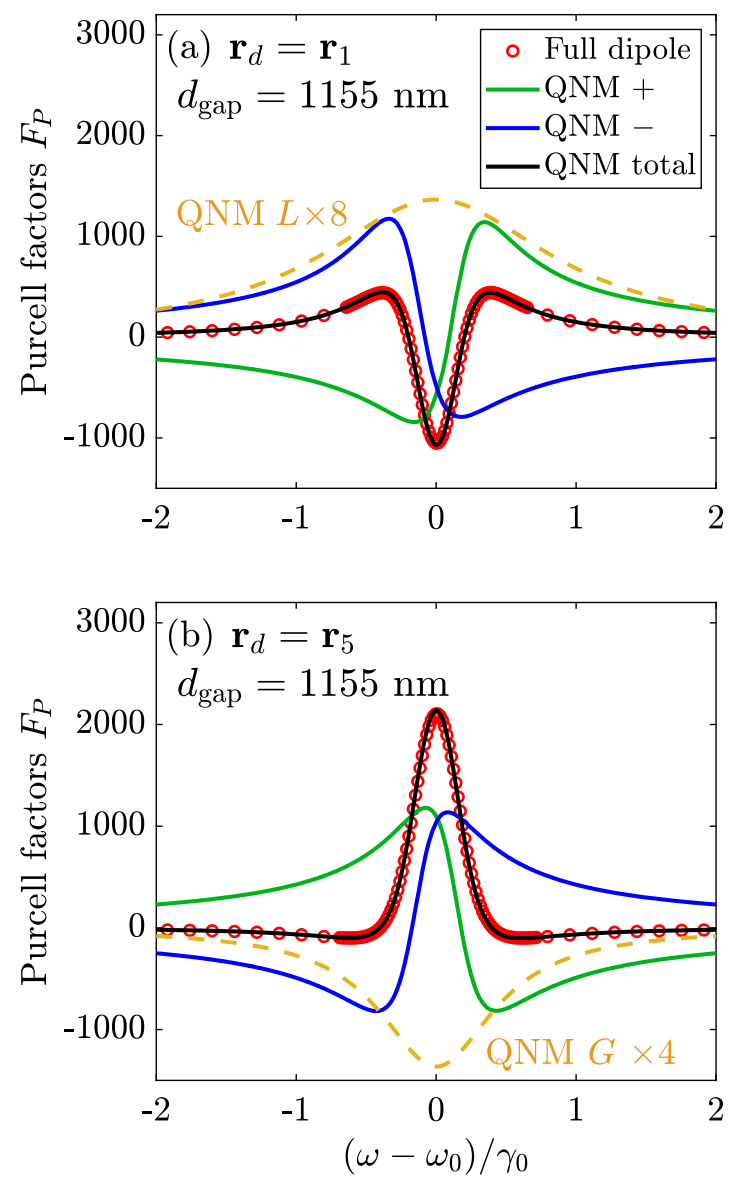

FIG. 4. (a) Purcell factors for the coupled resonators with gap distance $d_{\text {gap }}=1155 \mathrm{~nm}$, showing the analytical CMT solutions [(Eqs. (39) and (5)] for the hybrid QNM contributions as well as their sum. Also shown is the full dipole simulation with no approximations [red marker, Eq. (C1)] and the QNM contribution from a single-lossy resonator (multiplied by a constant for clarity). The dipole is placed at $\mathbf{r}_{d}=\mathbf{r}_{1}$ (10 nm away from the lossy resonator). (b) Similar to (a), but with a different dipole position $\mathbf{r}_{d}=\mathbf{r}_{5}, 10 \mathrm{~nm}$ away from the gain resonator.

where $A^{+}(\omega)=\omega /\left[2\left(\tilde{\omega}_{+}-\omega\right)\right], A^{-}(\omega)=\omega /\left[2\left(\tilde{\omega}_{-}-\omega\right)\right]$, with $\tilde{\omega}_{+}=\omega_{+}-i \gamma_{+}$and $\tilde{\omega}_{-}=\omega_{-}-i \gamma_{-}$. From this, we extract the imaginary part for use in Purcell's formula [100]:

$$
\begin{aligned}
& \operatorname{Im}\left[G_{z z}\left(\mathbf{r}_{d}, \mathbf{r}_{d}, \omega\right)\right] \\
& =\left[\cos 2 \phi^{+}\left(\mathbf{r}_{d}\right)+\frac{\omega_{+}-\omega}{\gamma_{+}} \sin 2 \phi^{+}\left(\mathbf{r}_{d}\right)\right]\left|\tilde{f}_{z}^{+}\left(\mathbf{r}_{d}\right)\right|^{2} L^{+}(\omega) \\
& \quad+\left[\cos 2 \phi^{-}\left(\mathbf{r}_{d}\right)+\frac{\omega_{-}-\omega}{\gamma_{-}} \sin 2 \phi^{-}\left(\mathbf{r}_{d}\right)\right]\left|\tilde{f}_{z}^{-}\left(\mathbf{r}_{d}\right)\right|^{2} L^{-}(\omega),
\end{aligned}
$$

where we introduce the modified Lorentzian line shape functions, 


$$
\begin{aligned}
L^{+}(\omega) & =\frac{\omega}{2} \frac{\gamma_{+}}{\left(\omega_{+}-\omega\right)^{2}+\gamma_{+}^{2}}, \\
L^{-}(\omega) & =\frac{\omega}{2} \frac{\gamma_{-}}{\left(\omega_{-}-\omega\right)^{2}+\gamma_{-}^{2}} .
\end{aligned}
$$

Similar expressions have been used to explain Fano resonances formed by elastic QNMs in coupled-cavity beams [100].

For comparison, the Green's functions for the singlemode bare QNMs are also given as

$$
\begin{aligned}
\operatorname{Im} & {\left[G_{z z}^{L}\left(\mathbf{r}_{d}, \mathbf{r}_{d}, \omega\right)\right] } \\
& =\left[\cos 2 \phi^{L}\left(\mathbf{r}_{d}\right)+\frac{\omega_{L}-\omega}{\gamma_{L}} \sin 2 \phi^{L}\left(\mathbf{r}_{d}\right)\right]\left|\tilde{f}_{z}^{L}\left(\mathbf{r}_{d}\right)\right|^{2} L^{L}(\omega),
\end{aligned}
$$

$$
\begin{aligned}
\operatorname{Im} & {\left[G_{z z}^{G}\left(\mathbf{r}_{d}, \mathbf{r}_{d}, \omega\right)\right] } \\
& =\left[\cos 2 \phi^{G}\left(\mathbf{r}_{d}\right)+\frac{\omega_{G}-\omega}{\gamma_{G}} \sin 2 \phi^{G}\left(\mathbf{r}_{d}\right)\right]\left|\tilde{f}_{z}^{G}\left(\mathbf{r}_{d}\right)\right|^{2} L^{G}(\omega),
\end{aligned}
$$

with modified Lorentzian line shape functions,

$$
\begin{aligned}
L^{L}(\omega) & =\frac{\omega}{2} \frac{\gamma_{L}}{\left(\omega_{L}-\omega\right)^{2}+\gamma_{L}^{2}}, \\
L^{G}(\omega) & =\frac{\omega}{2} \frac{\gamma_{G}}{\left(\omega_{G}-\omega\right)^{2}+\gamma_{G}^{2}},
\end{aligned}
$$

for the loss and gain resonator modes, and we redefine the QNMs as $\tilde{f}_{z}^{L}(\mathbf{r})=\left|\tilde{f}_{z}^{L}(\mathbf{r})\right| e^{i \phi^{L}(\mathbf{r})}$ and $\tilde{f}_{z}^{G}(\mathbf{r})=\left|\tilde{f}_{z}^{G}(\mathbf{r})\right| e^{i \phi^{G}(\mathbf{r})}$.

In Fig. 4(a), as mentioned above, we show the total Purcell factors for $d_{\text {gap }}=1155 \mathrm{~nm}$, as well as the hybrid mode contributions, and the lossy mode result on its own for comparison. First, the single-mode case (orange dashed curve, single-lossy resonator) shows a typical Lorentzian peak, which can be explained by the QNM phase contributions: $\cos \left[2 \phi^{L}\left(\mathbf{r}_{1}\right)\right]=1.0000$ and $\sin \left[2 \phi^{L}\left(\mathbf{r}_{1}\right)\right]=-0.0012$ (also shown in Table I). Applying these to Eq. (49) results in a typical Lorentzian-like line shape. Note since with QNM expansion, the imaginary part of the Green function with the presence of the cavity satisfies $\operatorname{ImG} \propto \omega$, while for background homogeneous medium, it satisfies $\operatorname{Im}_{\mathbf{B}} \propto \omega^{2}$, then the expected line shape (with no phase influence) has a trivial $\omega$ dependence; however, for the high- $Q$ resonances we consider, the simple QNM line shape is basically Lorentzian.

Next we focus on the hybrid modes (coupled-resonator case), where now $\cos \left[2 \phi^{+}\left(\mathbf{r}_{1}\right)\right]=0.1514, \sin \left[2 \phi^{+}\left(\mathbf{r}_{1}\right)\right]=$ -0.9885 , and $\cos \left[2 \phi^{-}\left(\mathbf{r}_{1}\right)\right]=0.1939, \quad \sin \left[2 \phi^{-}\left(\mathbf{r}_{1}\right)\right]=$ 0.9810 (also shown in Table I), which explain the highly non-Lorentzian line shapes for the separate contributions [two terms in Eq. (47), green solid curve and blue solid curve shown in Fig. 4(a)]. Then combining the weights for each term [from $\left|\tilde{f}_{z}^{+}\left(\mathbf{r}_{1}\right)\right|^{2}$ and $\left|\tilde{f}_{z}^{-}\left(\mathbf{r}_{1}\right)\right|^{2}$ as shown in Eq. (47)], we obtain negative Purcell factors in a wide range of frequencies [black solid curve in Fig. 4(a)].

Furthermore, these QNM phases result in positiondependent line shapes for the Purcell factors. Thus, when we change the dipole position from $\mathbf{r}_{d}=\mathbf{r}_{1}$ to $\mathbf{r}_{d}=\mathbf{r}_{5}$ (10 nm away from the gain cavity), the Purcell factor line shapes can change significantly, as shown in Fig. 4(b) (again for $d_{\text {gap }}=1155 \mathrm{~nm}$ ), where the total contribution (black solid curve) is found to be mainly net positive in a wide range of frequency and also show excellent agreement with the full dipole method (red circles). Once again, the contribution from $\tilde{\mathbf{f}}^{+}$and $\tilde{\mathbf{f}}^{-}$can be given separately, as shown with the green and blue solid curves. In addition, the Purcell factors for the single-gain cavity is shown as an orange dashed curve, which is net negative (and multiplied by 4 for a better graphical comparison).

As before, these line shapes can also be well explained from the QNM phase terms. For a single-gain cavity, one finds that $\cos \left[2 \phi^{G}\left(\mathbf{r}_{5}\right)\right]=1.0000$ and $\sin \left[2 \phi^{G}\left(\mathbf{r}_{5}\right)\right]=$ 0.0005 (also shown in Table I). However, with $\gamma_{G}<0$, $L^{G}(\omega)$ shows a negative Lorentzian line shape, yielding negative Purcell factors with the gain cavity only [orange dashed curve in Fig. 4(b)]. As for the coupled system, one can find $\cos \left[2 \phi^{+}\left(\mathbf{r}_{5}\right)\right]=0.1834, \sin \left[2 \phi^{+}\left(\mathbf{r}_{5}\right)\right]=0.9830$, and $\cos \left[2 \phi^{-}\left(\mathbf{r}_{5}\right)\right]=0.1620, \quad \sin \left[2 \phi^{-}\left(\mathbf{r}_{5}\right)\right]=-0.9868 \quad$ (also shown in Table I), which explain the non-Lorentzian line shapes for separate contribution [two terms in Eq. (47), green solid curve and blue solid curve shown in Fig. 4(b)]. Then, combining the weights for each term [from $\left|\tilde{f}_{z}^{+}\left(\mathbf{r}_{5}\right)\right|^{2}$ and $\left|\tilde{f}_{z}^{-}\left(\mathbf{r}_{5}\right)\right|^{2}$ in Eq. (47)], the mostly positive Purcell factors are obtained [black solid curve in Fig. 4(b)].

TABLE I. Phase contributions in the imaginary part of the QNM Green's function, which is used to explain the line shapes of the corresponding Purcell factors shown in Figs. 4 and 5.

\begin{tabular}{lcr}
\hline \hline & $\mathbf{r}_{d}=\mathbf{r}_{1}$ & $\mathbf{r}_{d}=\mathbf{r}_{5}$ \\
\hline QNM $L$ & $\cos \left(2 \phi^{\mathrm{L}}\right)=1.0000, \sin \left(2 \phi^{L}\right)=-0.0012$ & \\
\hline QNM $G$ & $\cos \left(2 \phi^{\mathrm{G}}\right)=1.0000, \sin \left(2 \phi^{\mathrm{G}}\right)=0.0005$ \\
\hline$d_{\text {gap }}=1155 \mathrm{~nm}$ & $\cos \left(2 \phi^{+}\right)=0.1514, \sin \left(2 \phi^{+}\right)=-0.9885$ & $\cos \left(2 \phi^{+}\right)=0.1834, \sin \left(2 \phi^{+}\right)=0.9830$ \\
& $\cos \left(2 \phi^{-}\right)=0.1939, \sin \left(2 \phi^{-}\right)=0.9810$ & $\cos \left(2 \phi^{-}\right)=0.1620, \sin \left(2 \phi^{-}\right)=-0.9868$ \\
\hline$d_{\text {gap }}=1160 \mathrm{~nm}$ & $\cos \left(2 \phi^{+}\right)=-1.0000, \sin \left(2 \phi^{+}\right)=0.0009$ & $\cos \left(2 \phi^{+}\right)=0.9999, \sin \left(2 \phi^{+}\right)=0.0101$ \\
& $\cos \left(2 \phi^{-}\right)=1.0000, \sin \left(2 \phi^{-}\right)=-0.0011$ & $\cos \left(2 \phi^{-}\right)=-0.9999, \sin \left(2 \phi^{-}\right)=-0.0129$ \\
\hline \hline
\end{tabular}



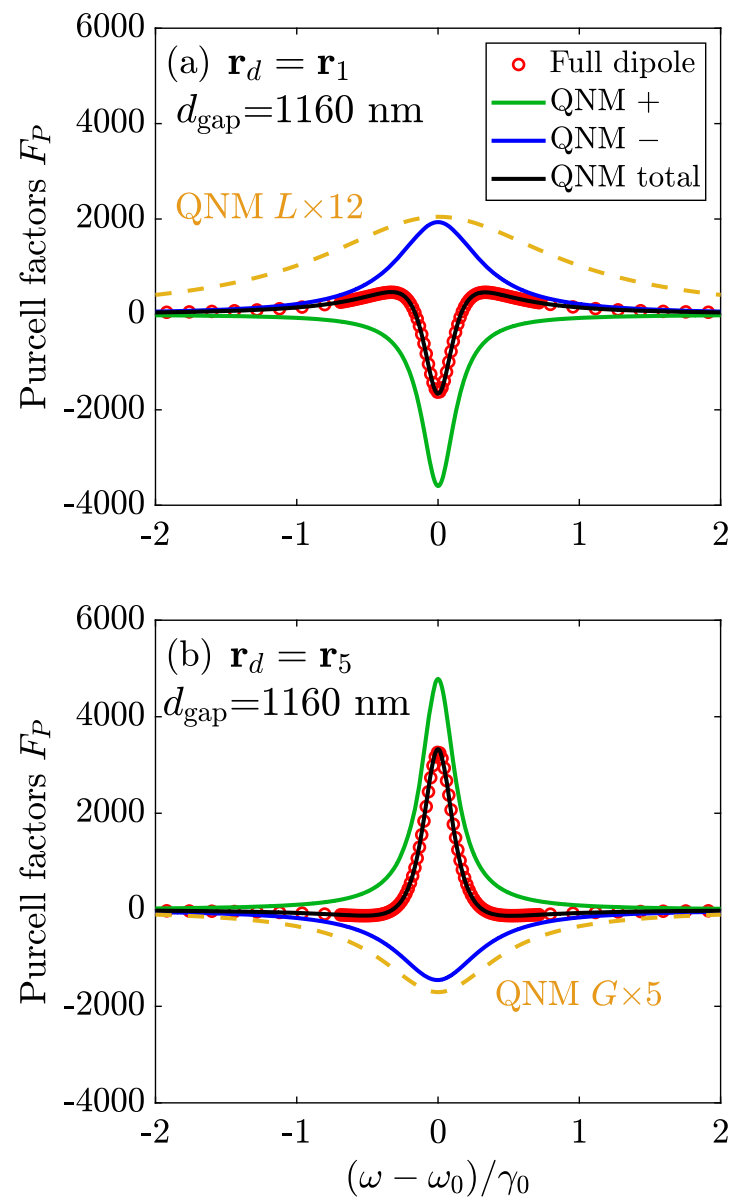

FIG. 5. Similar to Fig. 4, but with a different gap distance $d_{\text {gap }}=1160 \mathrm{~nm}$.

Similar to case with a gap distance $d_{\text {gap }}=1155 \mathrm{~nm}$, the Purcell factors with $d_{\text {gap }}=1160 \mathrm{~nm}$ for a dipole at $\mathbf{r}_{1}$ are shown in Fig. 5(a). Their separate contributions from QNM+ (green solid curve) and QNM- (blue solid curve) show Lorentzian line shapes, but with different linewidth since $\gamma_{+} \neq \gamma_{-}$[see Fig. 3(f)] and different signs due to $\cos \left[2 \phi^{+}\left(\mathbf{r}_{1}\right)\right]=-1.0000$ and $\cos \left[2 \phi^{-}\left(\mathbf{r}_{1}\right)\right]=1.0000$. Combing these contributions, the total contribution is found to be net negative in a wide range of frequencies. When the dipole is at $\mathbf{r}_{5}$, the Purcell factors are shown in Fig. 5(b), where the Lorentzian line shapes for separate contributions could also be explained by the QNM phases $\cos \left[2 \phi^{+}\left(\mathbf{r}_{5}\right)\right]=$ 0.9999 and $\cos \left[2 \phi^{-}\left(\mathbf{r}_{5}\right)\right]=-0.9999$. Considering their separate contributions, the total Purcell factors are now mostly positive. More details about the QNM phases are shown in Table I.

These QNM phases have a significant effect on the spectral line shapes of the Purcell factors, which can be further seen in Fig. 6(a) (for $d_{\text {gap }}=1155 \mathrm{~nm}$ ) and Fig. 6(b) (for $d_{\text {gap }}=1160 \mathrm{~nm}$ ), where the total Purcell factors are given for five different dipole positions as shown in Fig. 2. For better comparison, the Purcell factors at $\mathbf{r}_{2}, \mathbf{r}_{3}$, and $\mathbf{r}_{4}$ are multiplied by 10,300 , and 10 , respectively. Note, we do
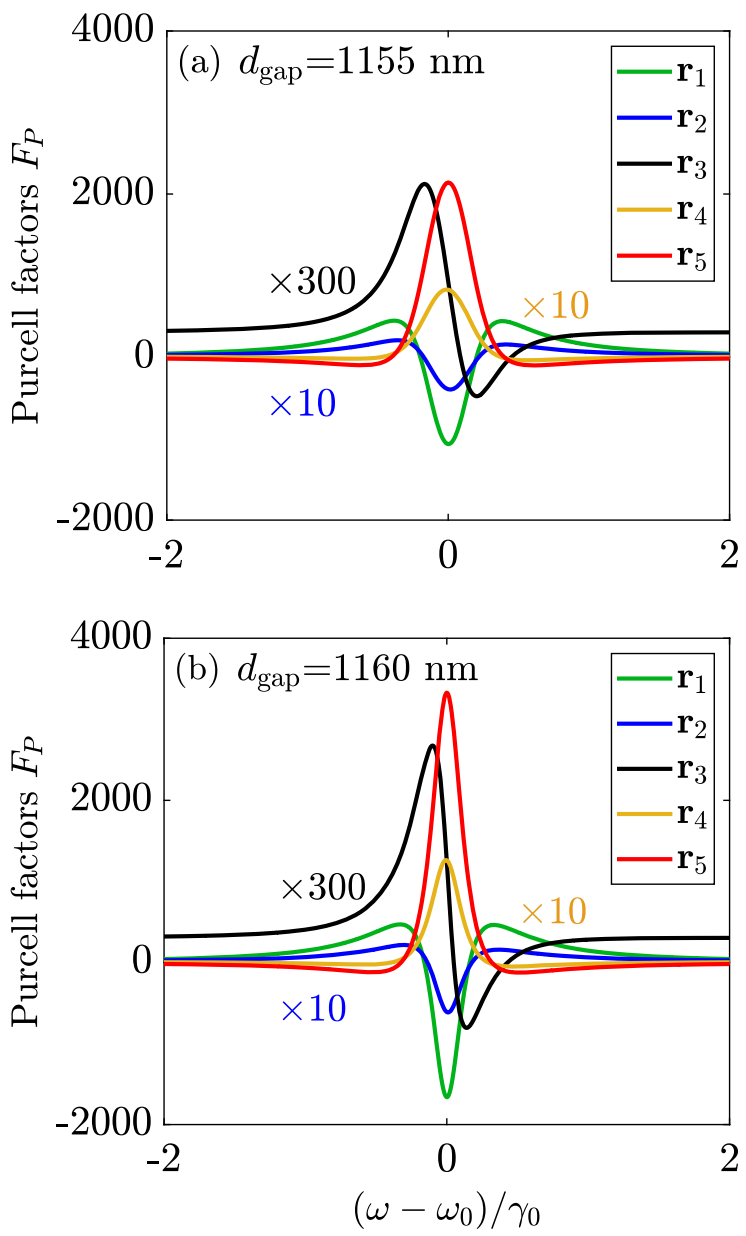

FIG. 6. Purcell factors at different dipole positions $\mathbf{r}_{d}=\mathbf{r}_{1} \sim \mathbf{r}_{5}$ (shown in Fig. 2), for two different gap separations (a) $d_{\text {gap }}=$ $1155 \mathrm{~nm}$, and (b) $d_{\text {gap }}=1160 \mathrm{~nm}$. Full dipole simulations are not shown for clarity, but yield the same quantitatively good fit as in the previous graphs.

not show the full dipole results here, as they are basically indistinguishable from the two QNM solutions, similar to the previous comparisons.

We stress that these negative Purcell factors are unphysical because the classical Purcell factor formulas, and Fermi's golden rule from purely dissipative media is no longer working with a gain medium [85], though the LDOS [proportional to $\left.\operatorname{Im}\left[\mathbf{G}\left(\mathbf{r}_{d}, \mathbf{r}_{d}, \omega\right)\right]\right]$ is correct, in agreement with the classical dipole result. To clarify, there is nothing unphysical about a negative LDOS in a medium that contains gain, but a negative Purcell factor clearly makes no sense as a normalized SE decay rate, since it would lead to an increase in excited-state population without bounds.

As a consequence of these results and using a rigorous quantum field theory, Ref. [85] discusses the breakdown of classical emission formulas in more detail and shows that the correct SE rates must be described fully quantum mechanically, where the quantum Purcell factors (modified 

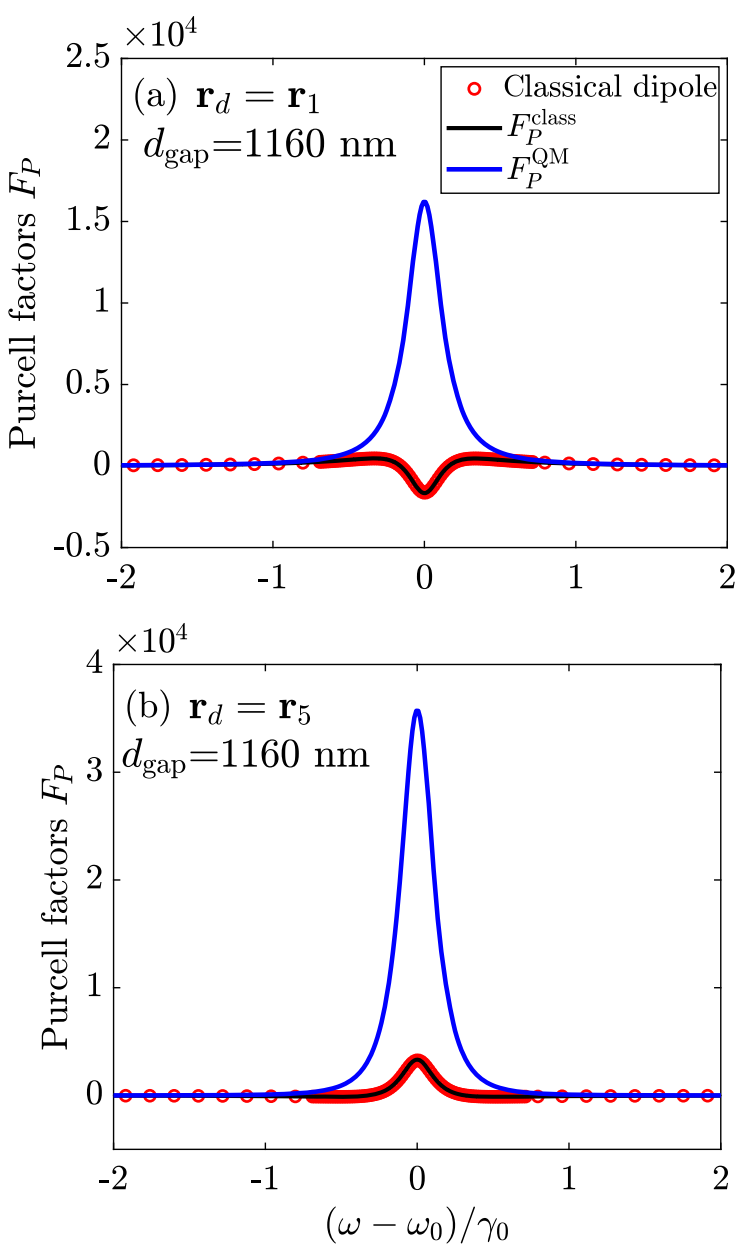

FIG. 7. Classical (established) Purcell factor $F_{P}^{\text {class }}=F_{P}$, [Eq. (5)] versus the quantum Purcell factor $F_{P}^{\mathrm{QM}}$ [Eq. (51)], which includes a gain correction that is not captured by the classical or semiclassical theories of spontaneous emission [85]. "Classical dipole" results (red circles) are from Eq. (C1), the same as those labeled as "Full dipole."

SE decay rates) are indeed net positive. In that case, the LDOS formula that connects to the classical Purcell factor $F_{P}^{\text {class }}$ [Eq. (5) together with Eq. (47)] is corrected by a positive gain-induced term, so that the (corrected) quantum Purcell factor is obtained from

$$
F_{P}^{\mathrm{QM}}=F_{P}^{\text {class }}+\frac{2|d|^{2} K^{\text {gain }}}{\hbar \epsilon_{0} \Gamma_{0}} .
$$

However, we also note that even in the case of amplifying and lossy media, the LDOS [contained in $F_{P}^{\text {class }}=$ $F_{P}$, Eq. (5)] is fundamentally related to the quantumvacuum fluctuations of the electric field commutator $\left\langle\operatorname{vac}\left|\left[\hat{\mathbf{E}}\left(\mathbf{r}_{d}\right), \hat{\mathbf{E}}^{\dagger}\left(\mathbf{r}_{d}\right)\right]\right| \operatorname{vac}\right\rangle$, and thus still reflects an important quantity in quantization of general electromagnetic media. Indeed, in a purely lossy case, $\langle\operatorname{vac}|\left[\hat{\mathbf{E}}\left(\mathbf{r}_{d}\right)\right.$, $\left.\hat{\mathbf{E}}^{\dagger}\left(\mathbf{r}_{d}\right)\right]|\operatorname{vac}\rangle$ coincides with $\left\langle\operatorname{vac}\left|\hat{\mathbf{E}}\left(\mathbf{r}_{d}\right) \hat{\mathbf{E}}^{\dagger}\left(\mathbf{r}_{d}\right)\right| \mathrm{vac}\right\rangle$, which is related to the cavity-enhanced $\mathrm{SE}$ rate of a quantum emitter at position $\mathbf{r}_{d}$.
Applying this correction to the dipole-microdisk-resonator (z-polarized dipole) system case, the quantum-correction factor $K^{\text {gain }}$ takes the explicit form

$$
\begin{aligned}
K^{\text {gain }}= & \left|A^{+}(\omega)\right|^{2}\left|\tilde{f}_{z}^{+}\left(\mathbf{r}_{d}\right)\right|^{2} I_{\text {gain }}^{+}+\left|A^{-}(\omega)\right|^{2}\left|\tilde{f}_{z}^{-}\left(\mathbf{r}_{d}\right)\right|^{2} I_{\text {gain }}^{-} \\
& +2 \operatorname{Re}\left[A^{+}(\omega) A^{-*}(\omega) \tilde{f}_{z}^{+}\left(\mathbf{r}_{d}\right) \tilde{f}_{z}^{-*}\left(\mathbf{r}_{d}\right) I_{\text {gain }}^{ \pm}\right]
\end{aligned}
$$

with diagonal integral expressions

$$
I_{\text {gain }}^{+(-)}=\left|\operatorname{Im}\left[\epsilon_{\text {gain }}\right]\right| \int_{A_{\text {gain }}} d \mathbf{r}\left|\mathbf{f}_{z}^{+(-)}(\mathbf{r})\right|^{2},
$$

and an overlap integral

$$
I_{\text {gain }}^{ \pm}=\left|\operatorname{Im}\left[\epsilon_{\text {gain }}\right]\right| \int_{A_{\text {gain }}} d \mathbf{r f}^{+}(\mathbf{r}) \cdot \mathbf{f}^{-*}(\mathbf{r}),
$$

where $\epsilon_{\text {gain }}=n_{\text {gain }}^{2}$ is the permittivity of the gain resonator, and $A_{\text {gain }}$ stands for the region within it. Despite leading to a net positive Purcell factor, this correction term also induces a drastic increase of the classical Purcell enhancement itself, as demonstrated in Fig. 7.

\section{Green's function propagators}

This section presents example Green's function propagator results, namely, $\left|\mathbf{G}\left(\mathbf{R}, \mathbf{r}_{d}, \omega\right)\right|$, which can be useful to relate to a range of experimental observables. For example, this function is required to model the spectrum at a point detector located at $\mathbf{R}$ emitted from a dipole at $\mathbf{r}_{d}$. Unlike the (classical) Purcell factor, it is a well-defined quantity for use in both classical and quantum field theory, and it is used frequently in the exploration of light-matter coupling regimes [107-109]. For example, considering an excitation dipole $\mathbf{d}$, the spectrum is $S(\mathbf{R}, \omega) \propto\left|\mathbf{G}\left(\mathbf{R}, \mathbf{r}_{d}, \omega\right) \cdot \mathbf{d}\right|^{2}$.

A far-field detection point is first chosen at $\mathbf{R}=\mathbf{R}_{1}=$ $[0,10] \mu \mathrm{m}$ (the origin of the coordinate system is at the gap center). The corresponding propagators $\left|G_{z z}\left(\mathbf{R}, \mathbf{r}_{d}, \omega\right)\right|$ (arbitrary units) for various dipole positions $\mathbf{r}_{d}$ are shown in Fig. 8(a) for $d_{\text {gap }}=1160 \mathrm{~nm}$. For comparison, the propagator for a single-lossy (-gain) cavity is also shown as green (red) dashed curve, where the dipole is placed at $\mathbf{r}_{d}=\mathbf{r}_{1}\left(\mathbf{r}_{5}\right)$. Furthermore, for the far-field detected point at $\mathbf{R}=\mathbf{R}_{2}=$ $[14.09,0] \mu \mathrm{m}$, the propagators are shown in Fig. 8(b).

To better explain the physics of the propagator line shape, we once more write out the two QNM expanded Green's function in terms of the hybrid modes,

$$
\begin{aligned}
G_{z z}\left(\mathbf{R}, \mathbf{r}_{d}, \omega\right) & \\
= & A^{+}(\omega) \tilde{f}_{z}^{+}(\mathbf{R}) \tilde{f}_{z}^{+}\left(\mathbf{r}_{d}\right)+A^{-}(\omega) \tilde{f}_{z}^{-}(\mathbf{R}) \tilde{f}_{z}^{-}\left(\mathbf{r}_{d}\right) \\
= & A^{+}(\omega) e^{i\left(\phi^{+}(\mathbf{R})+\phi^{+}\left(\mathbf{r}_{d}\right)\right)}\left|\tilde{f}_{z}^{+}(\mathbf{R})\right|\left|\tilde{f}_{z}^{+}\left(\mathbf{r}_{d}\right)\right| \\
& +A^{-}(\omega) e^{i\left(\phi^{-}(\mathbf{R})+\phi^{-}\left(\mathbf{r}_{d}\right)\right)}\left|\tilde{f}_{z}^{-}(\mathbf{R})\right|\left|\tilde{f}_{z}^{-}\left(\mathbf{r}_{d}\right)\right|,
\end{aligned}
$$



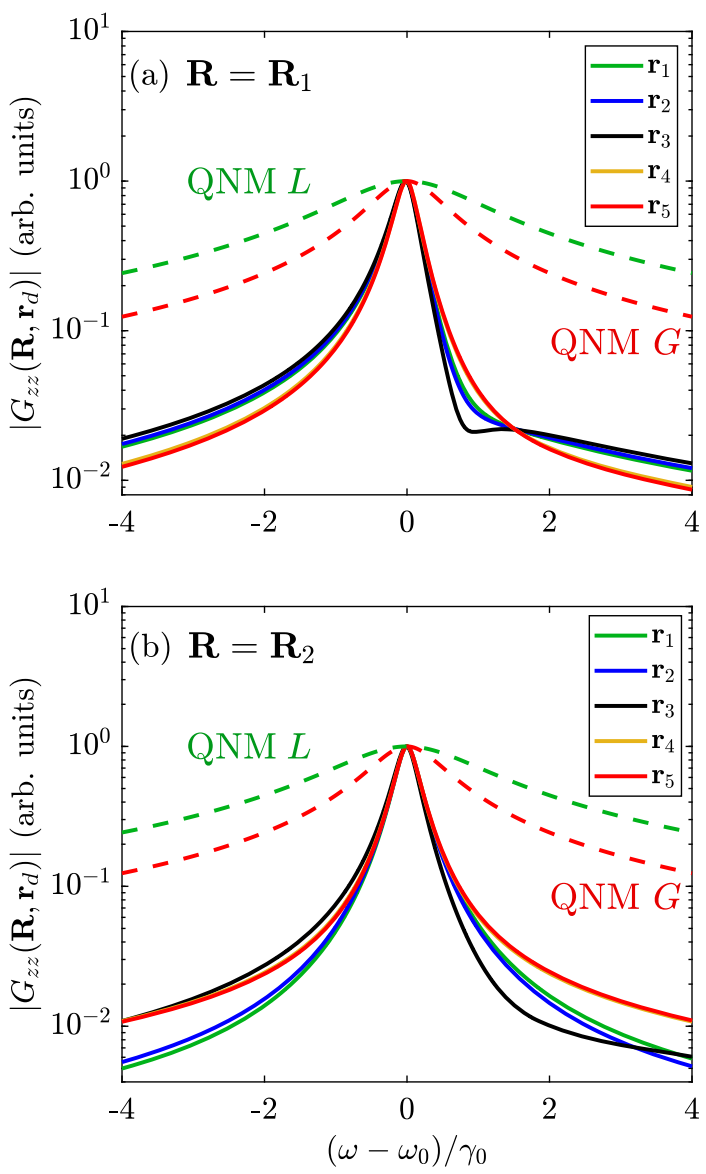

FIG. 8. Green's function propagator for the far-field detected point is at (a) $\mathbf{R}=\mathbf{R}_{1}=[0,10] \mu \mathrm{m}$ and (b) $\mathbf{R}=\mathbf{R}_{2}=$ $[14.09,0] \mu \mathrm{m}$ (which is $3.51 \mu \mathrm{m}$ away from the gain cavity) with $d_{\text {gap }}=1160 \mathrm{~nm}$. For QNM $L$ (loss), the dipole is at $\mathbf{r}_{d}=\mathbf{r}_{1}$, and for QNM $G$ (gain), it is at $\mathbf{r}_{d}=\mathbf{r}_{5}$. The results for $d_{\text {gap }}=1155 \mathrm{~nm}$ look similar, and we do not show them.

where again we can recognize non-Lorentzian features from the QNM phase terms, now from both the dipole position and the detector position. The detailed phases are shown in Table II. Note that the two-space-point Green's function has a range of uses for describing light-matter

TABLE II. Phase contributions from the QNMs in the propagator [Eq. (55)].

\begin{tabular}{lcr}
\hline \hline & \multicolumn{1}{c}{$\mathbf{R}=\mathbf{R}_{1}$} & $\mathbf{R}=\mathbf{R}_{2}$ \\
\hline QNM $L$ & $\cos \left[\phi^{L}(\mathbf{R})+\phi^{L}\left(\mathbf{r}_{d}\right)\right]=-0.6905$ & -0.9989 \\
$\mathbf{r}_{d}=\mathbf{r}_{1}$ & $\sin \left[\phi^{L}(\mathbf{R})+\phi^{L}\left(\mathbf{r}_{d}\right)\right]=0.7234$ & -0.0470 \\
\hline $\mathrm{QNM} G$ & $\cos \left[\phi^{G}(\mathbf{R})+\phi^{G}\left(\mathbf{r}_{d}\right)\right]=-0.7036$ & 0.2606 \\
$\mathbf{r}_{d}=\mathbf{r}_{5}$ & $\sin \left[\phi^{G}(\mathbf{R})+\phi^{G}\left(\mathbf{r}_{d}\right)\right]=0.7106$ & -0.9655 \\
\hline$d_{\text {gap }}=1160 \mathrm{~nm}$ & $\cos \left[\phi^{+}(\mathbf{R})+\phi^{+}\left(\mathbf{r}_{d}\right)\right]=-0.0815$ & 0.9760 \\
& $\sin \left[\phi^{+}(\mathbf{R})+\phi^{+}\left(\mathbf{r}_{d}\right)\right]=-0.9967$ & 0.2177 \\
$\mathbf{r}_{d}=\mathbf{r}_{1}$ & $\cos \left[\phi^{-}(\mathbf{R})+\phi^{-}\left(\mathbf{r}_{d}\right)\right]=-0.0575$ & -0.9784 \\
& $\sin \left[\phi^{-}(\mathbf{R})+\phi^{-}\left(\mathbf{r}_{d}\right)\right]=0.9983$ & -0.2070 \\
\hline \hline
\end{tabular}

interactions, including the description of photon transport $[107,110,111]$ and can be used to model collective effects with multiple emitters and dipoles.

\section{CONCLUSIONS}

We have introduced a powerful and highly accurate QNM approach to coupled loss-gain resonators and presented an accurate and intuitive CMT based on the photonic Green's function, which allows one to solve the coupled system efficiently with just the bare QNM solutions from the individual resonators. We also highlighted the failure of using a NM CMT approach when defining the general conditions for finding EPs.

For the SE response of embedded dipoles in these systems, we performed detailed calculations for coupled microdisk resonators. As well as finding Lorentzian-like and Lorentzian-squared-like spectral responses at the EP as a limiting case, consistent with other works, we showed much richer Purcell factor line shapes near EPs for various designs and spatial dipole positions, showing excellent agreement with QNM CMT and full dipole calculations. In particular, we show how the LDOS and common expression for the classical Purcell factors can also be negative in loss-gain media, even when the hybrid modes are both lossy $\left(\gamma_{\mu}>0\right)$. This is caused by a breakdown of Fermi's golden rule which incorrectly assumes that the SE rate is propositional to the (projected) LDOS [85]. In addition, we also show how the Green's function propagators (related to various experimental observables, such as the emitted spectrum) also take on rich non-Lorentzian features, which depend on the values of the QNM phases.

Apart from providing a detailed and intuitive formalism for understanding the classical mode properties of these complex coupled-resonator systems, our QNM formalism forms the basis for a rigorous quantum-optics approach in media with gain and loss, using new approaches with quantized QNMs [75-77], which has already lead to a revision of the usual photonic Fermi's golden rule for coupled loss and gain resonators [85], one in which the (correct) quantum-mechanical Purcell factor is always a positive quantity.

\section{ACKNOWLEDGMENTS}

We acknowledge funding from Queen's University, the Canadian Foundation for Innovation, the Natural Sciences and Engineering Research Council of Canada, and CMC Microsystems for the provision of COMSOL Multiphysics. We also acknowledge support from the Alexander von Humboldt Foundation through a Humboldt research grant. We thank Andreas Knorr for discussions and support and Thomas Christopoulos for discussions about perfectly matched layer (PML) properties in COMSOL. 


\section{APPENDIX A: QNM NORMALIZATION}

There are several general numerical approaches to obtaining normalized QNMs [72], including a dipoleexcitation technique in complex frequency [68], PML normalization [66], finite-domain normalization with a surface term $[73,74,86]$, finite-difference time-domain methods [89], Riesz-projection-based techniques [69], and Gaussian regularization [112]. In the main text, we use the dipole technique in complex frequency space [68], and more details are shown in Appendix A 1. A brief description of two alternative methods is also given in Appendices A 2 and A 3; numerically, we find all three approaches give the same normalized QNMs for the QNMs used in this work (within numerical precision).

\section{QNM normalization and numerically exact Green's function from a dipole source}

As shown in the main text, with CMT and the Green's function theory, only the bare mode solutions (for a singlelossy resonator or -gain resonator) are required. We employ an efficient dipole-scattering approach to obtain the uncoupled QNMs in complex frequency [68], where an out-of-plane line current (a point in 2D, red dot in Fig. 2, for a TM mode) is placed close to the lossy resonator, or the gain resonator. We can also use this approach for the coupled-resonator problem, which we also do to check the accuracy of the analytical CMT for the hybrid modes (see Appendix E).

The scattered field of this dipole at $\mathbf{r}_{0}$ is related to the $2 \mathrm{D}$ Green's function from

$$
\mathbf{E}^{\text {scatt }}(\mathbf{r}, \omega)=\mathbf{G}^{2 \mathrm{D}}\left(\mathbf{r}, \mathbf{r}_{0}, \omega\right) \cdot \frac{\mathbf{d}^{2 \mathrm{D}}}{\epsilon_{0}},
$$

where the units of the scattered field $\mathbf{E}^{\text {scatt }}, 2 \mathrm{D}$ Green's function $\mathbf{G}^{2 \mathrm{D}}$, and $2 \mathrm{D}$ dipole moment $\mathbf{d}^{2 \mathrm{D}}$ are, respectively, $\mathrm{V} / \mathrm{m}, \mathrm{m}^{-2}$, and $\mathrm{C}$.

Expanding the 2D Green's function with one QNM (dominating in the regime of interest), then

$$
\mathbf{G}^{2 \mathrm{D}}\left(\mathbf{r}, \mathbf{r}_{0}, \omega\right)=A_{c}(\omega) \tilde{\mathbf{f}}_{c}^{2 \mathrm{D}}(\mathbf{r}) \tilde{\mathbf{f}}_{c}^{2 \mathrm{D}}\left(\mathbf{r}_{0}\right),
$$

with $A_{c}(\omega)=\omega /\left[2\left(\tilde{\omega}_{c}-\omega\right)\right]$, so that

$$
\mathbf{E}^{\text {scatt }}(\mathbf{r}, \omega)=\frac{1}{\epsilon_{0}} A_{c}(\omega) \tilde{\mathbf{f}}_{c}^{2 \mathrm{D}}(\mathbf{r}) \tilde{\mathbf{f}}_{c}^{2 \mathrm{D}}\left(\mathbf{r}_{0}\right) \cdot \mathbf{d}^{2 \mathrm{D}} .
$$

Multiplying Eq. (A3) with $\mathbf{d}^{2 \mathrm{D}}$ and using $\mathbf{r}=\mathbf{r}_{0}$, then

$$
\mathbf{d}^{2 \mathrm{D}} \cdot \tilde{\mathbf{f}}_{c}^{2 \mathrm{D}}\left(\mathbf{r}_{0}\right)=\sqrt{\frac{\epsilon_{0} \mathbf{d}^{2 \mathrm{D}} \cdot \mathbf{E}^{\mathrm{scatt}}\left(\mathbf{r}_{0}, \omega\right)}{A_{c}(\omega)}} .
$$

Substituting this back into Eq. (A3), we obtain the 2D normalized QNM field as a function of space

$$
\begin{aligned}
& \tilde{\mathbf{f}}_{c}^{2 \mathrm{D}}(\mathbf{r})=\sqrt{\frac{\epsilon_{0}}{A_{c}(\omega) \mathbf{d}^{2 \mathrm{D}} \cdot \mathbf{E}^{\mathrm{scatt}}\left(\mathbf{r}_{0}, \omega\right)}} \mathbf{E}^{\mathrm{scatt}}(\mathbf{r}, \omega), \\
& =\sqrt{\frac{2 \epsilon_{0}\left(\tilde{\omega}_{c}-\omega\right)}{\omega \mathbf{d}^{2 \mathrm{D}} \cdot \mathbf{E}^{\text {scatt }}\left(\mathbf{r}_{0}, \omega\right)}} \mathbf{E}^{\text {scatt }}(\mathbf{r}, \omega) \text {. }
\end{aligned}
$$

The above QNM simulations are performed in the commercial COMSOL software [113], where the frequency in Eq. (A5) is set as $\omega=\left(1-10^{-7}\right) \times \tilde{\omega}_{c}$ [or $\left(1-10^{-6}\right) \times \tilde{\omega}_{c}$, $\left(1-10^{-8}\right) \times \tilde{\omega}_{c}$, adjusted with the quality factors], very close to the pole frequency. The computational domain (including PMLs) is around $804-814 \mu \mathrm{m}^{2}$ (various gap distance for microdisk resonators, where the maximum mesh element sizes are $0.1,40$, and $75 \mathrm{~nm}$ at the dipole point, inside and outside the 2D microdisks. To minimize boundary reflections, we use 15 layers to form the PMLs with a total thickness of $1.5 \mu \mathrm{m}$, which is found to be well converged numerically.

In addition, once the normalized QNMs are available, the corresponding effective mode area (with units $\mathrm{m}^{2}$ ), which is a function of the position, is obtained from

$$
A_{c}^{\mathrm{eff}}\left(\mathbf{r}_{0}\right)=\frac{1}{\epsilon\left(\mathbf{r}_{0}\right) \operatorname{Re}\left[\left(\tilde{\mathbf{f}}_{c}^{2 \mathrm{D}}\left(\mathbf{r}_{0}\right)\right)^{2}\right]} .
$$

The decay rates for 2D dipoles are as follows:

$\Gamma^{2 \mathrm{D}}\left(\mathbf{r}_{0}, \omega\right)=\frac{2}{\hbar \epsilon_{0}} \mathbf{d}^{2 \mathrm{D}} \cdot \operatorname{Im}\left\{\mathbf{G}^{2 \mathrm{D}}\left(\mathbf{r}_{0}, \mathbf{r}_{0}, \omega\right)\right\} \cdot \mathbf{d}^{2 \mathrm{D}}$,

$\Gamma_{0}^{2 \mathrm{D}}\left(\mathbf{r}_{0}, \omega\right)=\frac{2}{\hbar \epsilon_{0}} \mathbf{d}^{2 \mathrm{D}} \cdot \operatorname{Im}\left\{\mathbf{G}_{B}^{2 \mathrm{D}}\left(\mathbf{r}_{0}, \mathbf{r}_{0}, \omega\right)\right\} \cdot \mathbf{d}^{2 \mathrm{D}}$,

where $\operatorname{Im}\left\{\mathbf{G}_{B}\left(\mathbf{r}_{0}, \mathbf{r}_{0}, \omega\right)\right\}=\omega^{2} / 4 c^{2}$ for a $2 \mathrm{D}$ TM dipole, and the units of $\Gamma^{2 \mathrm{D}}$ are $1 /(\mathrm{s} \mathrm{m})$.

The corresponding Purcell factor is simply $[67,88]$

$$
F^{2 \mathrm{D}}\left(\mathbf{r}_{0}, \omega\right)=1+\frac{\Gamma^{2 \mathrm{D}}\left(\mathbf{r}_{0}, \omega\right)}{\Gamma_{0}^{2 \mathrm{D}}\left(\mathbf{r}_{0}, \omega\right)},
$$

and similar expressions are obtained for 3D systems.

\section{PML normalization}

The PML normalization [66,71,114-116] approach is another alternative way to get the normalized QNMs, which is given via

$$
\begin{aligned}
\left\langle\left\langle\tilde{\mathbf{f}}_{\mu}(\mathbf{r}) \mid \tilde{\mathbf{f}}_{\mu}(\mathbf{r})\right\rangle=\right. & \frac{1}{2 \epsilon_{0}} \int_{V}\left[\left.\epsilon_{0} \frac{\partial(\omega \epsilon(\mathbf{r}, \omega))}{\partial \omega}\right|_{\tilde{\omega}_{\mu}} \tilde{\mathbf{f}}_{\mu}(\mathbf{r}) \cdot \tilde{\mathbf{f}}_{\mu}(\mathbf{r})\right. \\
& \left.-\left.\mu_{0} \frac{\partial(\omega \mu(\mathbf{r}, \omega))}{\partial \omega}\right|_{\tilde{\omega}_{\mu}} \tilde{\mathbf{h}}_{\mu}(\mathbf{r}) \cdot \tilde{\mathbf{h}}_{\mu}(\mathbf{r})\right] d V=1,
\end{aligned}
$$

where $V$ is the whole numerical simulation region, which also includes the PML region. Next, one can divide the integral into two parts, 


$$
\begin{aligned}
\left\langle\left\langle\tilde{\mathbf{f}}_{\mu}(\mathbf{r}) \mid \tilde{\mathbf{f}}_{\mu}(\mathbf{r})\right\rangle=\right. & \frac{1}{2 \epsilon_{0}} \int_{V-V_{\mathrm{PML}}}\left[\left.\epsilon_{0} \frac{\partial(\omega \epsilon(\mathbf{r}, \omega))}{\partial \omega}\right|_{\tilde{\omega}_{\mu}} \tilde{\mathbf{f}}_{\mu}(\mathbf{r}) \cdot \tilde{\mathbf{f}}_{\mu}(\mathbf{r})-\left.\mu_{0} \frac{\partial(\omega \mu(\mathbf{r}, \omega))}{\partial \omega}\right|_{\tilde{\omega}_{\mu}} \tilde{\mathbf{h}}_{\mu}(\mathbf{r}) \cdot \tilde{\mathbf{h}}_{\mu}(\mathbf{r})\right] d V \\
& +\frac{1}{2 \epsilon_{0}} \int_{V_{\mathrm{PML}}}\left[\left.\epsilon_{0} \frac{\partial\left(\omega \epsilon_{\mathrm{PML}}(\mathbf{r}, \omega)\right)}{\partial \omega}\right|_{\tilde{\omega}_{\mu}} \tilde{\mathbf{f}}_{\mu}(\mathbf{r}) \cdot \tilde{\mathbf{f}}_{\mu}(\mathbf{r})-\left.\mu_{0} \frac{\partial\left(\omega \mu_{\mathrm{PML}}(\mathbf{r}, \omega)\right)}{\partial \omega}\right|_{\tilde{\omega}_{\mu}} \tilde{\mathbf{h}}_{\mu}(\mathbf{r}) \cdot \tilde{\mathbf{h}}_{\mu}(\mathbf{r})\right] d V=1,
\end{aligned}
$$

where the first part excludes the PML data, and the second part includes only the PML data. The term $\tilde{\mathbf{h}}_{\mu}(\mathbf{r})=$ $\boldsymbol{\nabla} \times \tilde{\mathbf{f}}_{\mu}(\mathbf{r}) / i \tilde{\omega}_{\mu} \mu_{0}$ is the magnetic QNM field and $V_{\mathrm{PML}}$ denotes the PML region. Outside the PML region, the fields are zero.

For a nondispersive and nonmagnetic material (not including the PML region), it becomes

$$
\begin{aligned}
\left\langle\left\langle\tilde{\mathbf{f}}_{\mu}(\mathbf{r}) \mid \tilde{\mathbf{f}}_{\mu}(\mathbf{r})\right\rangle=\right. & \frac{1}{2} \int_{V-V_{\mathrm{PML}}}\left[\epsilon(\mathbf{r}) \tilde{\mathbf{f}}_{\mu}(\mathbf{r}) \cdot \tilde{\mathbf{f}}_{\mu}(\mathbf{r})-\frac{\mu_{0}}{\epsilon_{0}} \tilde{\mathbf{h}}_{\mu}(\mathbf{r}) \cdot \tilde{\mathbf{h}}_{\mu}(\mathbf{r})\right] d V+\frac{1}{2 \epsilon_{0}} \int_{V_{\mathrm{PML}}}\left[\left.\epsilon_{0} \frac{\partial\left(\omega \epsilon_{\mathrm{PML}}(\mathbf{r}, \omega)\right)}{\partial \omega}\right|_{\tilde{\omega}_{\mu}} \tilde{\mathbf{f}}_{\mu}(\mathbf{r}) \cdot \tilde{\mathbf{f}}_{\mu}(\mathbf{r})\right. \\
& \left.-\left.\mu_{0} \frac{\partial\left(\omega \mu_{\mathrm{PML}}(\mathbf{r}, \omega)\right)}{\partial \omega}\right|_{\tilde{\omega}_{\mu}} \tilde{\mathbf{h}}_{\mu}(\mathbf{r}) \cdot \tilde{\mathbf{h}}_{\mu}(\mathbf{r})\right] d V=1,
\end{aligned}
$$

and note that extra care is needed for the PML region [second term in Eq. (A11)], though this contribution can be very small for certain problems and geometries. In general, there are several kinds of transformations performed in PMLs to minimize boundary reflections. The first one is using special permittivity $\epsilon_{\mathrm{PML}}(\mathbf{r}, \omega)$ and permeability $\mu_{\mathrm{PML}}(\mathbf{r}, \omega)$ values, which are not always available with some commercial software [71]. A second approach uses a coordinate transformation $[115,116]$, which is what we use here, with the built-in stretched-coordinate PML of COMSOL, where the coordinates are transferred from real space to the complex plane [117].

To verify that the dipole normalization technique and PML normalization are consistent with each other, we perform the norm calculation [Eq. (A11)] using the QNM fields from the dipole technique. We obtain $\left\langle\left\langle\tilde{\mathbf{f}}^{L}(\mathbf{r}) \mid \tilde{\mathbf{f}}^{L}(\mathbf{r})\right\rangle\right\rangle=1.0004-$ $0.0011 i \sim 1$ for the QNM of interest from the singlelossy WGM resonator with $n_{\text {loss }}=2+10^{-5} i$, and
$\left\langle\left\langle\tilde{\mathbf{f}}^{G}(\mathbf{r}) \mid \tilde{\mathbf{f}}^{G}(\mathbf{r})\right\rangle\right\rangle=0.9991+0.0006 i \sim 1$ for the QNM of interest from single-gain WGM resonator with $n_{\text {gain }}=$ $2-5 \times 10^{-6} i$. However, note that for our chosen PML geometry and resonator, the contribution from the PML is practically negligible for this problem of interest (high- $Q$ resonance).

Although the PML contributions are obviously small because of our high- $Q$ resonator modes, we also check that a normalization of approximately 1 is obtained for a very low- $Q$ metallic (gold) resonator similar to those studied in Ref. [89], yielding values of $\left\langle\left\langle\tilde{\mathbf{f}}^{\text {metal }}(\mathbf{r}) \mid \tilde{\mathbf{f}}^{\text {metal }}(\mathbf{r})\right\rangle=\right.$ $1.0020+0.0018 i \sim 1$.

\section{Finite-domain normalization with a surface term}

A third way to normalize QNMs is through a finitedomain normalization with an outgoing surface term [72$74,86]$. Assuming nonmagnetic media, the normalization takes the form $[72,86]$

$$
\begin{aligned}
\left.\left\langle\tilde{\mathbf{f}}_{\mu}(\mathbf{r}) \mid \tilde{\mathbf{f}}_{\mu}(\mathbf{r})\right\rangle\right\rangle= & \frac{1}{2 \epsilon_{0}} \int_{V}\left[\left.\epsilon_{0} \frac{\partial(\omega \epsilon(\mathbf{r}, \omega))}{\partial \omega}\right|_{\tilde{\omega}_{\mu}} \tilde{\mathbf{f}}_{\mu}(\mathbf{r}) \cdot \tilde{\mathbf{f}}_{\mu}(\mathbf{r})-\mu_{0} \tilde{\mathbf{h}}_{\mu}(\mathbf{r}) \cdot \tilde{\mathbf{h}}_{\mu}(\mathbf{r})\right] d V \\
& -\frac{i}{2 \epsilon_{0} \tilde{\omega}_{\mu}} \int_{\partial V}\left[\left[r \partial r \tilde{\mathbf{f}}_{\mu}(\mathbf{r})\right] \times \tilde{\mathbf{h}}_{\mu}(\mathbf{r})-\tilde{\mathbf{f}}_{\mu}(\mathbf{r}) \times\left[r \partial r \tilde{\mathbf{h}}_{\mu}(\mathbf{r})\right]\right] \cdot \hat{\mathbf{n}} d A=1 .
\end{aligned}
$$

Alternatively, in terms of only the electric field QNMs $[73,74,86]$,

$$
\left\langle\left\langle\tilde{\mathbf{f}}_{\mu}(\mathbf{r}) \mid \tilde{\mathbf{f}}_{\mu}(\mathbf{r})\right\rangle\right\rangle=\int_{V} \sigma\left(\mathbf{r}, \tilde{\omega}_{\mu}\right) \tilde{\mathbf{f}}_{\mu}(\mathbf{r}) \cdot \tilde{\mathbf{f}}_{\mu}(\mathbf{r}) d \mathbf{r}+\frac{1}{2 \tilde{k}_{\mu}^{2}} \int_{\partial V}\left[\tilde{\mathbf{f}}_{\mu}(\mathbf{r}) \cdot \partial s\left[r \partial r \tilde{\mathbf{f}}_{\mu}(\mathbf{r})\right]-r\left[\partial r \tilde{\mathbf{f}}_{\mu}(\mathbf{r})\right] \cdot\left[\partial s \tilde{\mathbf{f}}_{\mu}(\mathbf{r})\right]\right] d A=1,
$$

where

$$
\sigma\left(\mathbf{r}, \tilde{\omega}_{\mu}\right)=\left.\frac{1}{2 \omega} \frac{\partial\left[\omega^{2} \epsilon(\mathbf{r}, \omega)\right]}{\partial \omega}\right|_{\tilde{\omega}_{\mu}}
$$


and $\partial V$ represents the surface of the finite domain $V$ that surrounds all resonators, and $\partial s$ is the derivative in the normal direction of the surface $\partial V$. In this case, the integral region $V$ has a greater freedom of choice, but at the cost of adding a surface integral (though for 2D, we simply need to compute a simple surface integral and a line integral). For a nondispersive material, the normalization simplifies further to

$$
\begin{aligned}
\left\langle\left\langle\tilde{\mathbf{f}}_{\mu}(\mathbf{r}) \mid \tilde{\mathbf{f}}_{\mu}(\mathbf{r})\right\rangle\right\rangle= & \int_{V} \epsilon(\mathbf{r}) \tilde{\mathbf{f}}_{\mu}(\mathbf{r}) \cdot \tilde{\mathbf{f}}_{\mu}(\mathbf{r}) d \mathbf{r} \\
& +\frac{1}{2 \tilde{k}_{\mu}^{2}} \int_{\partial V}\left[\tilde{\mathbf{f}}_{\mu}(\mathbf{r}) \cdot \partial s\left[r \partial r \tilde{\mathbf{f}}_{\mu}(\mathbf{r})\right]\right. \\
& \left.-r\left[\partial r \tilde{\mathbf{f}}_{\mu}(\mathbf{r})\right] \cdot\left[\partial s \tilde{\mathbf{f}}_{\mu}(\mathbf{r})\right]\right] d A=1 .
\end{aligned}
$$

For completeness, we also checked our normalized QNMs with this technique and obtain $\left\langle\left\langle\tilde{\mathbf{f}}^{L}(\mathbf{r}) \mid \tilde{\mathbf{f}}^{L}(\mathbf{r})\right\rangle=1.0004-\right.$ $0.0011 i \sim 1$ for the single bare QNM from the single-lossy WGM resonator with $n_{\text {loss }}=2+10^{-5} i$, and $\left\langle\left\langle\tilde{\mathbf{f}}^{G}(\mathbf{r}) \mid \tilde{\mathbf{f}}^{G}(\mathbf{r})\right\rangle\right\rangle=$ $0.9991+0.0006 i \sim 1$ for the single bare QNM from the single-gain WGM resonator with $n_{\text {gain }}=2-5 \times 10^{-6} i$.
Again, we note for the resonators we are working with the contribution from the line integral is negligible; thus, we obtain basically the same answer as the PML normalization. As a further check, we also try the above norm with two smaller domains with area of 579 and $497 \mu \mathrm{m}^{2}$ and obtain the same numerical results as above.

\section{APPENDIX B: DEGENERATE QNMS FOR MICRODISK RESONATORS}

The WGM resonator naturally supports degenerate modes because of circular symmetry. For a specific azimuthal mode number $m$, the degenerate counterpropagating modes are [104-106] $E_{\mathrm{CW}}(\mathbf{r}, \phi)=E(\mathbf{r}) e^{(-i m \phi)}$ with CW direction and $E_{\mathrm{CCW}}(\mathbf{r}, \phi)=E(\mathbf{r}) e^{(i m \phi)}$ with $\mathrm{CCW}$ direction; for a TM mode, we have only the $z$ component for the electric fields. Linear combinations of these fields result in standing waves [104-106], such as a symmetric standing mode $E_{S}=E_{\mathrm{CW}}+$ $E_{\mathrm{CCW}} \propto \cos (m \phi)$ and an antisymmetric standing mode $E_{\mathrm{AS}}=E_{\mathrm{CW}}-E_{\mathrm{CCW}} \propto \sin (m \phi)$. These standing waves can of course also be normalized and can be expanded to obtain the QNM Green's function. (a) $\operatorname{Re}\left[\tilde{f}_{z}^{L}\right]$

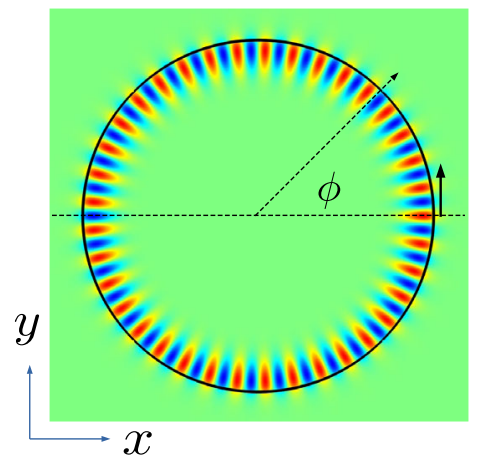

(b) $\operatorname{Re}\left[\tilde{f}_{z}^{M, 1}\right]$

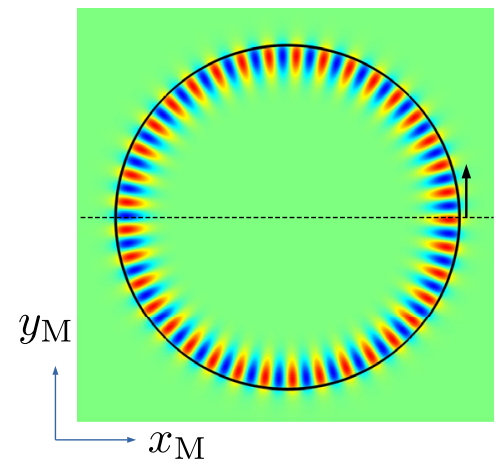

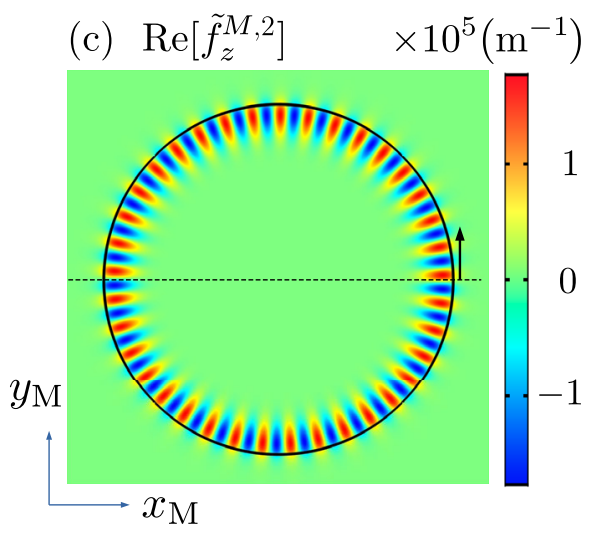

(f)

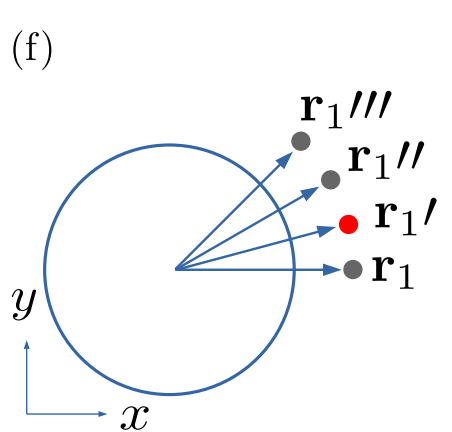

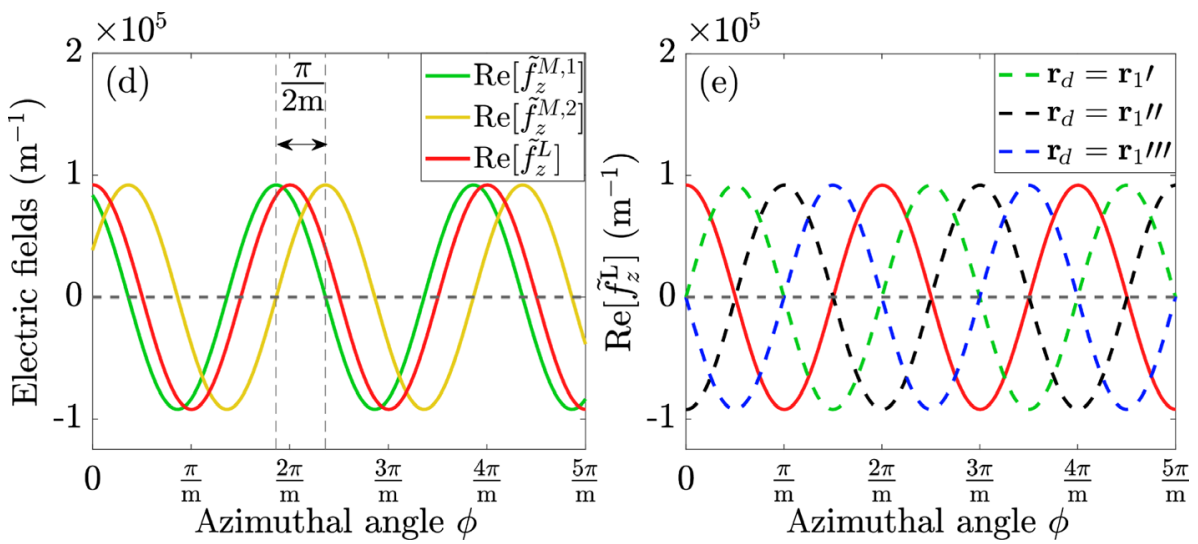

FIG. 9. Spatial profile of the degenerate QNMs for a single-lossy resonator. (a) QNM obtained with a general QNM approach [Eq. (A5)] [it is the same as in Fig. 3(a)], where only one of the degenerate standing-wave modes is excited, which is with a cos $(m \phi)$ dependence, considering $\phi=0$ at the positive $x$ axis. (b),(c) Normalized [Eq. (B1)] degenerate modes 1 and 2 from the eigenfrequency solver using COMSOL, where the boundary condition is with PML. The horizontal dashed lines in (a)-(c) label the $x$ axis with their own coordinates. (d) The fields at the disk edge along vertical arrow direction (CCW direction) [in (a)-(c)]. (e) QNMs [Eq. (A5)], same as in (a) but when the dipole is moved to a different position, as shown in (f). 
Using the direct eigenfrequency solver (where the boundary condition is PML) in COMSOL, one can obtain a pair of unnormalized standing-mode distributions $\tilde{\mathbf{f}}_{\mathrm{un}}^{M, 1 / M, 2}$. This is not used generally as a QNM solver since it is not so robust as a nonlinear eigenvalue solver, but the results appear to be reasonable for the high- $Q$ modes of interest in this work.

For example, one can use PML normalization to obtain the relevant QNM from $(i=1,2)$

$$
\tilde{\mathbf{f}}^{M, i}(\mathbf{r})=\frac{\tilde{\mathbf{f}}_{\mathrm{un}}^{M, i}(\mathbf{r})}{\sqrt{\left\langle\left\langle\tilde{\mathbf{f}}_{\mathrm{un}}^{M, i}(\mathbf{r}) \mid \tilde{\mathbf{f}}_{\mathrm{un}}^{M, i}(\mathbf{r})\right\rangle_{\mathrm{PML}}\right.}},
$$

where $\left\langle\left\langle\tilde{\mathbf{f}}_{\mathrm{un}}^{M, i}(\mathbf{r}) \mid \tilde{\mathbf{f}}_{\mathrm{un}}^{M, i}(\mathbf{r})\right\rangle\right\rangle_{\mathrm{PML}}$ takes the form of Eq. (A11). For a single-lossy WGM resonator with refractive index $n_{\text {loss }}=2+10^{-5} i$, the normalized distribution $\operatorname{Re}\left[\tilde{f}_{z}^{M, 1 / M, 2}\right]$ [Eq. (B1)] is shown in Figs. 9(b) and 9(c). To better check their differences, we show them along the edge of the disk (equator) along the small vertical arrow direction (CCW direction) [see Figs. 9(b) and 9(c)] in Fig. 9(d) (green solid and orange solid curves). One can find their antinode positions are off by $\pi / 2 m(m=37)$ corresponding to the difference between $\cos (m \phi)$ and $\sin (m \phi)$. However, their absolute phases are offset, since these are related to where $\phi=0$ is defined in the coordinate system $\left(x_{M}, y_{M}\right)$, with the eigenfrequency solver.

In contrast, with the dipole-excitation technique [68], only one of the two degenerate standing-wave modes is excited, as shown in Fig. 9(a) [which is the same as Fig. 3(a) in the main text], where the dipole is placed at $\mathbf{r}_{d}=\mathbf{r}_{1}$. The field $\operatorname{Re}\left[\tilde{f}_{z}^{L}\right]$ along the equator is also shown in Figs. 9(d) and 9(e) (red solid curve), which is with the form $\cos (m \phi)$ considering $\phi=0$ corresponds to the positive $x$ axis. Moreover, with the dipole technique, one can control the phases accurately by setting the dipole at different locations, as they naturally excite the same standing wave defined from that position. For example, if the dipole is placed at a position corresponding to $\phi \prime=\pi / 2 m$ $\left(\mathbf{r}_{d}=\mathbf{r}_{1} \prime\right), \quad \phi^{\prime \prime}=2 \pi / 2 m \quad\left(\mathbf{r}_{d}=\mathbf{r}_{1} \prime \prime\right), \quad$ or $\quad \phi^{\prime \prime \prime}=3 \pi / 2 m$ $\left(\mathbf{r}_{d}=\mathbf{r}_{1} / \prime \prime\right)$ (the distance between the dipoles and the tangent of the disk surface remains at $10 \mathrm{~nm}$ ) [see schematic in Fig. 9(f)], then the same QNM is obtained with a simple shift in phase; these are shown in Fig. 9(e) (green dashed, black dashed, and blue dashed curves), with the phase terms $\cos \left(m \phi-m \phi^{\prime}\right)=\cos (m \phi-\pi / 2), \cos (m \phi-m \phi \prime \prime)=$ $\cos (m \phi-2 \pi / 2)$, and $\cos (m \phi-m \phi \prime \prime \prime)=\cos (m \phi-3 \pi / 2)$ (considering $\phi=0$ is defined at the positive $x$ axis).

\section{APPENDIX C: FULL NUMERICAL DIPOLE SIMULATIONS}

To check the validity of the Purcell factors from the QNMs and also the CMT Green's function solutions (for microdisks), we compare these directly with the numerical results from a full dipole method (namely, with no modal approximations), which is obtained as follows:
$F_{P}^{\text {num }, 2 \mathrm{D}}\left(\mathbf{r}_{0}, \omega\right)=\frac{\int_{L_{c}} \hat{\mathbf{n}} \cdot \mathbf{S}_{\text {dipole, total }}(\mathbf{r}, \omega) d L_{c}}{\int_{L_{c}} \hat{\mathbf{n}} \cdot \mathbf{S}_{\text {dipole, background }}(\mathbf{r}, \omega) d L_{c}}$,

where $L_{c}$ is a small circle (with radius $1 \mathrm{~nm}$ ) surrounding the point current (for the TM mode), and $\hat{\mathbf{n}}$ is a unit vector normal to $L_{c}$ pointing outward. The vector $\mathbf{S}(\mathbf{r}, \omega)$ is the Poynting vector at this small circle, and the subscripts "total" and "background" represent the case with and without the resonator, respectively.

\section{APPENDIX D: CONFIRMING THE ACCURACY OF THE QNMS FOR A SINGLE-LOSSY RESONATOR OR A SINGLE-GAIN RESONATOR}

In order to compare with the coupled-resonator results and to confirm the accuracy of a single QNM approach for the single resonators, we show the results for single-lossy
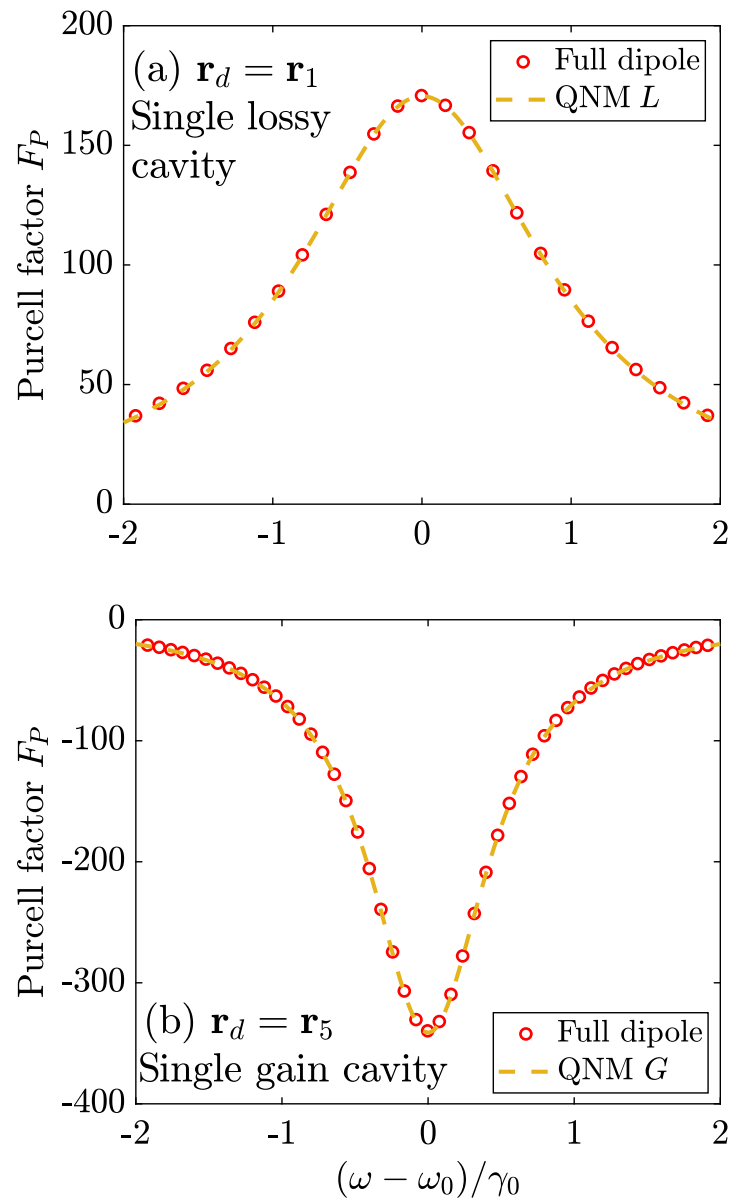

FIG. 10. Purcell factors for (a) single-lossy cavity with $n_{\text {loss }}=$ $2+10^{-5} i$ and (b) single-gain cavity with $n_{\text {gain }}=2-5 \times 10^{-6} i$ from single QNMs [Eqs. (5) or (A8)] and full numerical dipole method [Eq. (C1)], where excellent agreement is obtained in both cases. The dipole is located $10 \mathrm{~nm}$ away from the single-lossy cavity or single-gain cavity. The Purcell factors from the singlelossy cavity are net positive, while the Purcell factors from the single-gain cavity are net negative. 


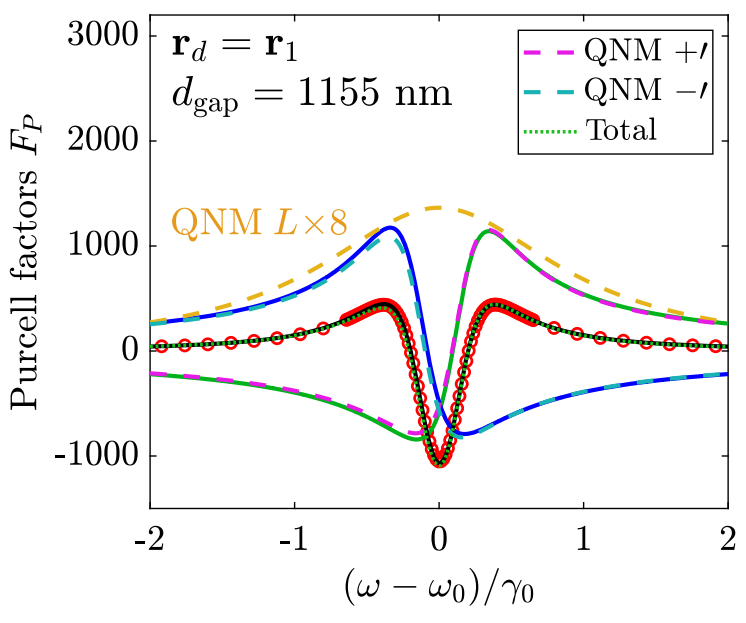

FIG. 11. Purcell factors from the direct QNM approach for the coupled-resonators example shown in Fig. 4(a) of the main text. For comparison, all original curves (analytical CMT results and full dipole results) in Fig. 4(a) remain the same. With the direct QNM approach (when the dipole is located at $\mathbf{r}_{d}=\mathbf{r}_{1}$ ), there are two dominant modes, QNM + । and QNM - I. Their separate contributions to the total Purcell factors are shown as a dashed magenta curve and a dashed cyan curve, and the total one is shown as a green dotted curve. They show good agreement with the CMT results and full dipole results; i.e., the contribution from QNM+ [green solid curve, see Fig. 4(a)] and QNM + / (dashed magenta curve) agrees very well, as does the contribution from QNM- (blue solid curve) and QNM - I (dashed cyan curve); the CMT and two direct QNM totals are practically indistinguishable (black solid curve, green dotted curve; red circles show the full dipole results).

cavity with $n_{\text {loss }}=2+10^{-5} i$ or single-gain cavity with $n_{\text {gain }}=2-5 \times 10^{-6} i$. Using the dipole technique described above [Eq. (A5)], the QNMs (real parts) $\operatorname{Re}\left[\tilde{f}_{z}^{L}\right]$ and $\operatorname{Re}\left[\tilde{f}_{z}^{G}\right]$ are shown in Figs. 3(a) and 3(b) separately. The dipole is put $10 \mathrm{~nm}$ away from the single-lossy cavity or single-gain cavity.

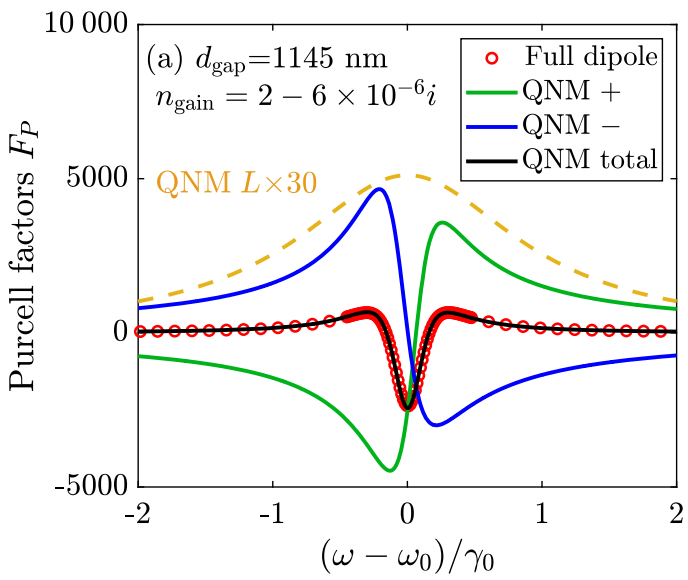

The corresponding Purcell factors are shown in Fig. 10, which show quantitative agreement with full dipole method. The Purcell factors from the single-lossy cavity are net positive and those from the single-gain cavity are net negative. Note that these results are also shown as dashed curves in Figs. 4 and 5 in the main text but multiplied by a constant for better comparison with the results from the coupled system.

\section{APPENDIX E: DIRECT QNM APPROACH FOR COUPLED LOSS RESONATORS AND GAIN RESONATORS}

Naturally, one can also solve the coupled system with a QNM approach directly instead of using CMT after the bare solutions are known. In Fig. 11, we show their comparison for the specific example shown in Fig. 4(a) of the main text. For comparison, all original curves (analytical CMT results and full dipole results) on Fig. 4(a) remain the same. With the dipole-excitation QNM approach (where the dipole is at $\mathbf{r}_{d}=\mathbf{r}_{1}$ ), there are two dominant modes $\mathrm{QNM}+\prime$ and QNM - / (the hybrid modes). Their separate contributions to the Purcell factors are shown as a magenta dashed curve and cyan dashed curve, and the total one is shown as a green dotted curve. There is very good agreement with the CMT results and the direct $\mathrm{QNM}$ results $(\mathrm{QNM}+$ versus $\mathrm{QNM}+$, QNM- versus QNM - I, and their total contributions), as well as quantitatively good agreement between the direct QNM results and the full dipole simulations (green dotted curve and red circles).

\section{APPENDIX F: ADDITIONAL LOSS-GAIN RESONATOR EXAMPLES WITH DIFFERENT GAIN COEFFICIENTS}

Finally, we also show the Purcell factors for two additional loss-gain resonator examples for different amounts of gain. The refractive index for the lossy resonator is fixed at

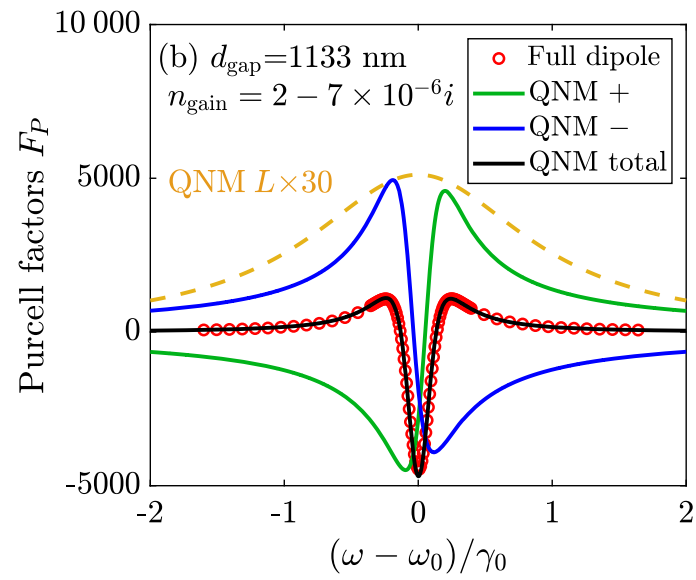

FIG. 12. Purcell factors for coupled loss-gain resonators with (a) $n_{\text {gain }}=2-6 \times 10^{-6} i$ and (b) $n_{\text {gain }}=2-7 \times 10^{-6} i$, while $n_{\text {loss }}=$ $2+10^{-5} i$ is the same as in the main text for both cases. The dipole is placed at $\mathbf{r}_{1}$. Similar to the main text results, once again very good agreement is obtained between with the semianalytic QNM theory and the full (classical) dipole results. 
$n_{\text {loss }}=2+10^{-5} i$, as in the main text. When $n_{\text {gain }}=$ $2-6 \times 10^{-6} i$, the Purcell factors with a gap distance $d_{\text {gap }}=$ $1145 \mathrm{~nm}$ (close to the lossy EP) for a dipole at $\mathbf{r}_{1}(10 \mathrm{~nm}$ away from the lossy cavity) are shown in Fig. 12(a), which show very good agreement with the full dipole method. Again, we see that negative Purcell factors are obtained over a wide frequency range. The separate contributions from $\tilde{\mathbf{f}}^{+}$and $\tilde{\mathbf{f}}^{-}$are also given. For better comparison, the Purcell factors with a single-lossy cavity are shown as an orange dashed curve (the dipole is at $\mathbf{r}_{1}$ ), which is net positive and multiplied by 30 for clarity.

Similarly, the corresponding results for the case with $n_{\text {gain }}=2-7 \times 10^{-6} i$ are shown in Fig. 12(b), where the gap distance is $d_{\text {gap }}=1133 \mathrm{~nm}$. Excellent agreement with full dipole results is also obtained. The absolute values of the negative Purcell factors increase here mainly because they are closer to the EP and the gap distance is smaller.

[1] C. Raabe and D.-G. Welsch, QED in Arbitrary Linear Media: Amplifying Media, Eur. Phys. J. Spec. Top. 160, 371 (2008).

[2] T. Søndergaard and B. Tromborg, General Theory for Spontaneous Emission in Active Dielectric Microstructures: Example of a Fiber Amplifier, Phys. Rev. A 64, 033812 (2001).

[3] C. M. Bender and S. Boettcher, Real Spectra in Non-Hermitian Hamiltonians Having $\mathcal{P} \mathcal{T}$ Symmetry, Phys. Rev. Lett. 80, 5243 (1998).

[4] C. M. Bender, S. Boettcher, and P. N. Meisinger, PT-Symmetric Quantum Mechanics, J. Math. Phys. (N.Y.) 40, 2201 (1999).

[5] G. Lévai and M. Znojil, Systematic Search for $\mathcal{P} \mathcal{T}$-Symmetric Potentials with Real Energy Spectra, J. Phys. A 33, 7165 (2000).

[6] C. M. Bender, M. V. Berry, and A. Mandilara, Generalized PT symmetry and Real Spectra, J. Phys. A 35, L467 (2002).

[7] C. M. Bender, D. C. Brody, and H. F. Jones, Complex Extension of Quantum Mechanics, Phys. Rev. Lett. 89, 270401 (2002).

[8] A. Mostafazadeh, Pseudo-Hermiticity versus PT Symmetry: The Necessary Condition for the Reality of the Spectrum of a Non-Hermitian Hamiltonian, J. Math. Phys. (N.Y.) 43, 205 (2002).

[9] C. M. Bender, D. C. Brody, and H. F. Jones, Must a Hamiltonian Be Hermitian? Am. J. Phys. 71, 1095 (2003).

[10] C. M. Bender, Making Sense of Non-Hermitian Hamiltonians, Rep. Prog. Phys. 70, 947 (2007).

[11] R. El-Ganainy, K. G. Makris, D. N. Christodoulides, and Z. H. Musslimani, Theory of Coupled Optical PTSymmetric Structures, Opt. Lett. 32, 2632 (2007).

[12] K. G. Makris, R. El-Ganainy, D. N. Christodoulides, and Z. H. Musslimani, Beam Dynamics in $\mathcal{P} \mathcal{T}$ Symmetric Optical Lattices, Phys. Rev. Lett. 100, 103904 (2008).

[13] S. Klaiman, U. Günther, and N. Moiseyev, Visualization of Branch Points in $\mathcal{P} \mathcal{T}$-Symmetric Waveguides, Phys. Rev. Lett. 101, 080402 (2008).
[14] A. Guo, G. J. Salamo, D. Duchesne, R. Morandotti, M. Volatier-Ravat, V. Aimez, G. A. Siviloglou, and D. N. Christodoulides, Observation of $\mathcal{P} \mathcal{T}$-Symmetry Breaking in Complex Optical Potentials, Phys. Rev. Lett. 103, 093902 (2009).

[15] S. Longhi, Bloch Oscillations in Complex Crystals with $\mathcal{P} \mathcal{T}$ Symmetry, Phys. Rev. Lett. 103, 123601 (2009).

[16] A. Mostafazadeh, Spectral Singularities of Complex Scattering Potentials and Infinite Reflection and Transmission Coefficients at Real Energies, Phys. Rev. Lett. 102, 220402 (2009).

[17] C. E. Rüter, K. G. Makris, R. El-Ganainy, D. N. Christodoulides, M. Segev, and D. Kip, Observation of Parity-Time Symmetry in Optics, Nat. Phys. 6, 192 (2010).

[18] T. Kottos, Broken Symmetry Makes Light Work, Nat. Phys. 6, 166 (2010).

[19] S. Longhi, Optical Realization of Relativistic NonHermitian Quantum Mechanics, Phys. Rev. Lett. 105, 013903 (2010).

[20] H. Benisty, A. Lupu, and A. Degiron, Transverse Periodic $\mathcal{P} \mathcal{T}$ Symmetry for Modal Demultiplexing in Optical Waveguides, Phys. Rev. A 91, 053825 (2015).

[21] V. V. Konotop, J. Yang, and D. A. Zezyulin, Nonlinear

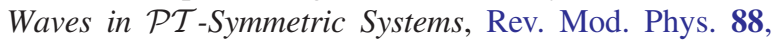
035002 (2016).

[22] L. Feng, R. El-Ganainy, and L. Ge, Non-Hermitian Photonics Based on Parity-Time Symmetry, Nat. Photonics 11, 752 (2017).

[23] S. Longhi, Parity-Time Symmetry Meets Photonics: A New Twist in Non-Hermitian Optics, Europhys. Lett. 120, 64001 (2017).

[24] A. Lupu, V. V. Konotop, and H. Benisty, Optimal $\mathcal{P} \mathcal{T}$-Symmetric Switch Features Exceptional Point, Sci. Rep. 7, 13299 (2017).

[25] R. El-Ganainy, K. G. Makris, M. Khajavikhan, Z. H. Musslimani, S. Rotter, and D. N. Christodoulides, NonHermitian Physics and PT Symmetry, Nat. Phys. 14, 11 (2018).

[26] L. Jin, Parity-Time-Symmetric Coupled Asymmetric Dimers, Phys. Rev. A 97, 012121 (2018).

[27] F. Morozko, A. Novitsky, and A. Karabchevsky, Modal Purcell Factor in $\mathcal{P} \mathcal{T}$-Symmetric Waveguides, Phys. Rev. B 102, 155303 (2020).

[28] M. V. Berry, Physics of Non-Hermitian Degeneracies, Czech. J. Phys. 54, 1039 (2004).

[29] W. D. Heiss, Exceptional Points of Non-Hermitian Operators, J. Phys. A 37, 2455 (2004).

[30] W. D. Heiss, The Physics of Exceptional Points, J. Phys. A 45, 444016 (2012).

[31] K. Ding, G. Ma, M. Xiao, Z. Q. Zhang, and C. T. Chan, Emergence, Coalescence, and Topological Properties of Multiple Exceptional Points and Their Experimental Tealization, Phys. Rev. X 6, 021007 (2016).

[32] M.-A. Miri and A. Alù, Exceptional Points in Optics and Photonics, Science 363, eaar7709 (2019).

[33] C. Chen, L. Jin, and R.-B. Liu, Sensitivity of Parameter Estimation near the Exceptional Point of a Non-Hermitian System, New J. Phys. 21, 083002 (2019).

[34] L. Jin, H. C. Wu, B.-B. Wei, and Z. Song, Hybrid Exceptional Point Created from Type-III Dirac Point, Phys. Rev. B 101, 045130 (2020). 
[35] H.-Z. Chen, T. Liu, H.-Y. Luan, R.-J. Liu, X.-Y. Wang, X.-F. Zhu, Y.-B. Li, Z.-M. Gu, S.-J. Liang, H. Gao, L. Lu, L. Ge, S. Zhang, J. Zhu, and R.-M. Ma, Revealing the Missing Dimension at an Exceptional Point, Nat. Phys. 16, 571 (2020).

[36] Z. Lin, H. Ramezani, T. Eichelkraut, T. Kottos, H. Cao, and D. N. Christodoulides, Unidirectional Invisibility Induced by $\mathcal{P} \mathcal{T}$-Symmetric Periodic Structures, Phys. Rev. Lett. 106, 213901 (2011).

[37] L. Feng, Y.-L. Xu, W. S. Fegadolli, M.-H. Lu, J. E. B. Oliveira, V. R. Almeida, Y.-F. Chen, and A. Scherer, Experimental Demonstration of a Unidirectional Reflectionless Parity-Time Metamaterial at Optical Frequencies, Nat. Mater. 12, 108 (2013).

[38] B. Peng, Ş. K. Özdemir, F. Lei, F. Monifi, M. Gianfreda, G. L. Long, S. Fan, F. Nori, C. M. Bender, and L. Yang, Parity-Time-Symmetric Whispering-Gallery Microcavities, Nat. Phys. 10, 394 (2014).

[39] L. Chang, X. Jiang, S. Hua, C. Yang, J. Wen, L. Jiang, G. Li, G. Wang, and M. Xiao, Parity-Time Symmetry and Variable Optical Isolation in Active-Passive-Coupled Microresonators, Nat. Photonics 8, 524 (2014).

[40] L. Jin and Z. Song, Incident Direction Independent Wave Propagation and Unidirectional Lasing, Phys. Rev. Lett. 121, 073901 (2018).

[41] A. A. Zyablovsky, A. P. Vinogradov, A. A. Pukhov, A. V. Dorofeenko, and A. A. Lisyansky, PT-Symmetry in Optics, Phys. Usp. 57, 1063 (2014).

[42] J. Doppler, A. A. Mailybaev, J. Böhm, U. Kuhl, A. Girschik, F. Libisch, T. J. Milburn, P. Rabl, N. Moiseyev, and S. Rotter, Dynamically Encircling an Exceptional Point for Asymmetric Mode Switching, Nature (London) 537, 76 (2016).

[43] H. Xu, D. Mason, L. Jiang, and J. G. E. Harris, Topological Energy Transfer in an Optomechanical System with Exceptional Points, Nature (London) 537, 80 (2016).

[44] D. Heiss, Circling Exceptional Points, Nat. Phys. 12, 823 (2016).

[45] W. Chen, Ş. K. Özdemir, G. Zhao, J. Wiersig, and L. Yang, Exceptional Points Enhance Sensing in an Optical Microcavity, Nature (London) 548, 192 (2017).

[46] W. Chen, J. Zhang, B. Peng, Ş. K. Özdemir, X. Fan, and L. Yang, Parity-Time-Symmetric Whispering-Gallery Mode Nanoparticle Sensor, Photonics Res. 6, A23 (2018).

[47] S. Longhi, $\mathcal{P} \mathcal{T}$-Symmetric Laser Absorber, Phys. Rev. A 82, 031801(R) (2010).

[48] Y. D. Chong, L. Ge, and A. D. Stone, $\mathcal{P} \mathcal{T}$-Symmetry Breaking and Laser-Absorber Modes in Optical Scattering Systems, Phys. Rev. Lett. 106, 093902 (2011).

[49] Y. Sun, W. Tan, H.-Q. Li, J. Li, and H. Chen, Experimental Demonstration of a Coherent Perfect Absorber with PT Phase Transition, Phys. Rev. Lett. 112, 143903 (2014).

[50] L. Jin, P. Wang, and Z. Song, Unidirectional Perfect Absorber, Sci. Rep. 6, 32919 (2016).

[51] M. Brandstetter, M. Liertzer, C. Deutsch, P. Klang, J. Schöberl, H. E. Türeci, G. Strasser, K. Unterrainer, and S. Rotter, Reversing the Pump Dependence of a Laser at an Exceptional Point, Nat. Commun. 5, 4034 (2014).
[52] L. Feng, Z. J. Wong, R.-M. Ma, Y. Wang, and X. Zhang, Single-Mode Laser by Parity-Time Symmetry Breaking, Science 346, 972 (2014).

[53] H. Hodaei, M.-A. Miri, M. Heinrich, D. N. Christodoulides, and M. Khajavikhan, Parity-Time-Symmetric Microring Lasers, Science 346, 975 (2014).

[54] B. Peng, Ş. K. Özdemir, S. Rotter, H. Yilmaz, M. Liertzer, F. Monifi, C. M. Bender, F. Nori, and L. Yang, LossInduced Suppression and Revival of Lasing, Science 346, 328 (2014).

[55] H. A. Haus and W. Huang, Coupled-Mode Theory, Proc. IEEE 79, 1505 (1991).

[56] S. Fan, W. Suh, and J.D. Joannopoulos, Temporal Coupled-Mode Theory for the Fano Resonance in Optical Resonators, J. Opt. Soc. Am. A 20, 569 (2003).

[57] A. Artar, A. A. Yanik, and H. Altug, Directional Double Fano Resonances in Plasmonic Hetero-Oligomers, Nano Lett. 11, 3694 (2011).

[58] R. Yang, Q. Fu, Y. Fan, W. Cai, K. Qiu, W. Zhang, and F. Zhang, Active Control of EIT-like Response in a SymmetryBroken Metasurface with Orthogonal Electric Dipolar Resonators, Photonics Res. 7, 955 (2019).

[59] J.-H. Park, A. Ndao, W. Cai, L. Hsu, A. Kodigala, T. Lepetit, Y.-H. Lo, and B. Kanté, Symmetry-BreakingInduced Plasmonic Exceptional Points and Nanoscale Sensing, Nat. Phys. 16, 462 (2020).

[60] H. M. Lai, P. T. Leung, K. Young, P. W. Barber, and S. C. Hill, Time-Independent Perturbation for Leaking Electromagnetic Modes in Open Systems with Application to Resonances in Microdroplets, Phys. Rev. A 41, 5187 (1990).

[61] P. T. Leung, S. Y. Liu, and K. Young, Completeness and Orthogonality of Quasinormal Modes in Leaky Optical Cavities, Phys. Rev. A 49, 3057 (1994).

[62] P. T. Leung, S. Y. Liu, S. S. Tong, and K. Young, TimeIndependent Perturbation Theory for Quasinormal Modes in Leaky Optical Cavities, Phys. Rev. A 49, 3068 (1994).

[63] P. T. Leung and K. M. Pang, Completeness and TimeIndependent Perturbation of Morphology-Dependent Resonances in Dielectric Spheres, J. Opt. Soc. Am. B 13, 805 (1996).

[64] K. M. Lee, P. T. Leung, and K. M. Pang, Dyadic Formulation of Morphology-Dependent Resonances. I. Completeness Relation, J. Opt. Soc. Am. B 16, 1409 (1999).

[65] P. T. Kristensen, C. Van Vlack, and S. Hughes, Generalized Effective Mode Volume for Leaky Optical Cavities, Opt. Lett. 37, 1649 (2012).

[66] C. Sauvan, J. P. Hugonin, I. S. Maksymov, and P. Lalanne, Theory of the Spontaneous Optical Emission of Nanosize Photonic and Plasmon Resonators, Phys. Rev. Lett. 110, 237401 (2013).

[67] P. T. Kristensen and S. Hughes, Modes and Mode Volumes of Leaky Optical Cavities and Plasmonic Nanoresonators, ACS Photonics 1, 2 (2014).

[68] Q. Bai, M. Perrin, C. Sauvan, J.-P. Hugonin, and P. Lalanne, Efficient and Intuitive Method for the Analysis of Light Scattering by a Resonant Nanostructure, Opt. Express 21, 27371 (2013).

[69] L. Zschiedrich, F. Binkowski, N. Nikolay, O. Benson, G. Kewes, and S. Burger, Riesz-Projection-Based Theory of 
Light-Matter Interaction in Dispersive Nanoresonators, Phys. Rev. A 98, 043806 (2018).

[70] F. Alpeggiani, N. Parappurath, E. Verhagen, and L. Kuipers, Quasinormal-Mode Expansion of the Scattering Matrix, Phys. Rev. X 7, 021035 (2017).

[71] P. Lalanne, W. Yan, K. Vynck, C. Sauvan, and J.-P. Hugonin, Light Interaction with Photonic and Plasmonic Resonances, Laser Photonics Rev. 12, 1700113 (2018).

[72] P. T. Kristensen, K. Herrmann, F. Intravaia, and K. Busch, Modeling Electromagnetic Resonators Using Quasinormal Modes, Adv. Opt. Photonics 12, 612 (2020).

[73] E. A. Muljarov, W. Langbein, and R. Zimmermann, Brillouin-Wigner Perturbation Theory in Open Electromagnetic Systems, Europhys. Lett. 92, 50010 (2010).

[74] E. A. Muljarov and W. Langbein, Exact Mode Volume and Purcell Factor of Open Optical Systems, Phys. Rev. B 94, 235438 (2016).

[75] S. Franke, S. Hughes, M. K. Dezfouli, P. T. Kristensen, K. Busch, A. Knorr, and M. Richter, Quantization of Quasinormal Modes for Open Cavities and Plasmonic Cavity Quantum Electrodynamics, Phys. Rev. Lett. 122, 213901 (2019).

[76] S. Franke, J. Ren, S. Hughes, and M. Richter, FluctuationDissipation Theorem and Fundamental Photon Commutation Relations in Lossy Nanostructures Using Quasinormal Modes, Phys. Rev. Research 2, 033332 (2020).

[77] S. Franke, M. Richter, J. Ren, A. Knorr, and S. Hughes, Quantized Quasinormal-Mode Description of Nonlinear Cavity-QED Effects from Coupled Resonators with a Fano-like Resonance, Phys. Rev. Research 2, 033456 (2020).

[78] B. Vial and Y. Hao, A Coupling Model for Quasi-Normal Modes of Photonic Resonators, J. Opt. 18, 115004 (2016).

[79] P. T. Kristensen, J. R. de Lasson, M. Heuck, N. Gregersen, and J. Mørk, On the Theory of Coupled Modes in Optical Cavity-Waveguide Structures, J. Lightwave Technol. 35, 4247 (2017).

[80] K. G. Cognée, Hybridization of Open Photonic Resonators, Ph. D. dissertation, University of Amsterdam, 2020.

[81] C. Tao, J. Zhu, Y. Zhong, and H. Liu, Coupling Theory of Quasinormal Modes for Lossy and Dispersive Plasmonic Nanoresonators, Phys. Rev. B 102, 045430 (2020).

[82] G. Yoo, H.-S. Sim, and H. Schomerus, Quantum Noise and Mode Nonorthogonality in Non-Hermitian $\mathcal{P} \mathcal{T}$-Symmetric Optical Resonators, Phys. Rev. A 84, 063833 (2011).

[83] A. Pick, B. Zhen, O. D. Miller, C. W. Hsu, F. Hernandez, A. W. Rodriguez, M. Soljačić, and S. G. Johnson, General Theory of Spontaneous Emission near Exceptional Points, Opt. Express 25, 12325 (2017).

[84] M. Khanbekyan and J. Wiersig, Decay Suppression of Spontaneous Emission of a Single Emitter in a High-Q Cavity at Exceptional Points, Phys. Rev. Research 2, 023375 (2020).

[85] S. Franke, J. Ren, M. Richter, A. Knorr, and S. Hughes, Fermi's Golden Rule for Spontaneous Emission in Absorptive and Amplifying Media, Phys. Rev. Lett. 127, 013602 (2021).

[86] P. T. Kristensen, R.-C. Ge, and S. Hughes, Normalization of Quasinormal Modes in Leaky Optical Cavities and Plasmonic Resonators, Phys. Rev. A 92, 053810 (2015).
[87] R.-C. Ge, P. T. Kristensen, J. F. Young, and S. Hughes, Quasinormal Mode Approach to Modelling Light-Emission and Propagation in Nanoplasmonics, New J. Phys. 16, 113048 (2014).

[88] P. Anger, P. Bharadwaj, and L. Novotny, Enhancement and Quenching of Single-Molecule Fluorescence, Phys. Rev. Lett. 96, 113002 (2006).

[89] R.-C. Ge and S. Hughes, Design of an Efficient Single Photon Source from a Metallic Nanorod Dimer: A QuasiNormal Mode Finite-Difference Time-Domain Approach, Opt. Lett. 39, 4235 (2014).

[90] A. R. Cowan and J. F. Young, Optical Bistability Involving Photonic Crystal Microcavities and Fano Line Shapes, Phys. Rev. E 68, 046606 (2003).

[91] J. Ren, S. Franke, A. Knorr, M. Richter, and S. Hughes, Near-Field to Far-Field Transformations of Optical Quasinormal Modes and Efficient Calculation of Quantized Quasinormal Modes for Open Cavities and Plasmonic Resonators, Phys. Rev. B 101, 205402 (2020).

[92] S. Hughes, S. Franke, C. Gustin, M. K. Dezfouli, A. Knorr, and M. Richter, Theory and Limits of On-Demand SinglePhoton Sources Using Plasmonic Resonators: A Quantized Quasinormal Mode Approach, ACS Photonics 6, 2168 (2019).

[93] P. T. Kristensen, J. R. de Lasson, and N. Gregersen, Calculation, Normalization, and Perturbation of Quasinormal Modes in Coupled Cavity-Waveguide Systems, Opt. Lett. 39, 6359 (2014).

[94] B. Peng, Ş. K. Özdemir, M. Liertzer, W. Chen, J. Kramer, H. Yilmaz, J. Wiersig, S. Rotter, and L. Yang, Chiral Modes and Directional Lasing at Exceptional Points, Proc. Natl. Acad. Sci. U.S.A. 113, 6845 (2016).

[95] S. Sunada, Enhanced Response of Non-Hermitian Photonic Systems near Exceptional Points, Phys. Rev. A 97, 043804 (2018).

[96] Z. Lin, A. Pick, M. Lončar, and A. W. Rodriguez, Enhanced Spontaneous Emission at Third-Order Dirac Exceptional Points in Inverse-Designed Photonic Crystals, Phys. Rev. Lett. 117, 107402 (2016).

[97] A. Pick, Z. Lin, W. Jin, and A. W. Rodriguez, Enhanced Nonlinear Frequency Conversion and Purcell Enhancement at Exceptional Points, Phys. Rev. B 96, 224303 (2017).

[98] J. R. de Lasson, P. T. Kristensen, J. Mørk, and N. Gregersen, Semianalytical Quasi-Normal Mode Theory for the Local Density of States in Coupled Photonic Crystal Cavity-Waveguide Structures, Opt. Lett. 40, 5790 (2015).

[99] M. K. Dezfouli, R. Gordon, and S. Hughes, Modal Theory of Modified Spontaneous Emission of a Quantum Emitter in a Hybrid Plasmonic Photonic-Crystal Cavity System, Phys. Rev. A 95, 013846 (2017).

[100] A.-W. El-Sayed and S. Hughes, Quasinormal-Mode Theory of Elastic Purcell Factors and Fano Resonances of Optomechanical Beams, Phys. Rev. Research 2, 043290 (2020).

[101] D. Pellegrino, D. Balestri, N. Granchi, M. Ciardi, F. Intonti, F. Pagliano, A. Yu. Silov, F. W. Otten, T. Wu, K. Vynck, P. Lalanne, A. Fiore, and M. Gurioli, NonLorentzian Local Density of States in Coupled Photonic 
Crystal Cavities Probed by Near-and Far-Field Emission, Phys. Rev. Lett. 124, 123902 (2020).

[102] G. C. Righini, Y. Dumeige, P. Féron, M. Ferrari, G. Nunzi Conti, D. Ristic, and S. Soria, Whispering Gallery Mode Microresonators: Fundamentals and Applications, Nuovo Cimento 34, 435 (2011).

[103] G. Schunk, J. U. Fürst, M. Förtsch, D. V. Strekalov, U. Vogl, F. Sedlmeir, H. G. L. Schwefel, G. Leuchs, and C. Marquardt, Identifying Modes of Large WhisperingGallery Mode Resonators from the Spectrum and Emission Pattern, Opt. Express 22, 30795 (2014).

[104] A. Mazzei, S. Götzinger, L. de S. Menezes, G. Zumofen, O. Benson, and V. Sandoghdar, Controlled Coupling of Counterpropagating Whispering-Gallery Modes by a Single Rayleigh Scatterer: A Classical Problem in a Quantum Optical Light, Phys. Rev. Lett. 99, 173603 (2007).

[105] I. Teraoka and S. Arnold, Resonance Shifts of Counterpropagating Whispering-Gallery Modes: Degenerate Perturbation Theory and Application to Resonator Sensors with Axial Symmetry, J. Opt. Soc. Am. B 26, 1321 (2009).

[106] K. G. Cognée, H. M. Doeleman, P. Lalanne, and A. F. Koenderink, Cooperative Interactions between NanoAntennas in a High-Q Cavity for Unidirectional Light Sources, Light Sci. Appl. 8, 115 (2019).

[107] M. Wubs, L. G. Suttorp, and A. Lagendijk, MultipleScattering Approach to Interatomic Interactions and Superradiance in Inhomogeneous Dielectrics, Phys. Rev. A 70, 053823 (2004).

[108] C. Van Vlack, P. T. Kristensen, and S. Hughes, Spontaneous Emission Spectra and Quantum Light-Matter Interactions from a Strongly Coupled Quantum Dot Metal-Nanoparticle System, Phys. Rev. B 85, 075303 (2012).
[109] R.-C. Ge, C. Van Vlack, P. Yao, J. F. Young, and S. Hughes, Accessing Quantum Nanoplasmonics in a Hybrid Quantum Dot-Metal Nanosystem: Mollow Triplet of a Quantum Dot near a Metal Nanoparticle, Phys. Rev. B 87, 205425 (2013).

[110] P. T. Kristensen, J. Mørk, P. Lodahl, and S. Hughes, Decay Dynamics of Radiatively Coupled Quantum Dots in Photonic Crystal Slabs, Phys. Rev. B 83, 075305 (2011).

[111] R.-C. Ge and S. Hughes, Quantum Dynamics of Two Quantum Dots Coupled through Localized Plasmons: An Intuitive and Accurate Quantum Optics Approach Using Quasinormal Modes, Phys. Rev. B 92, 205420 (2015).

[112] B. Stout, R. Colom, N. Bonod, and R. C. McPhedran, Spectral Expansions of Open and Dispersive Optical Systems: Gaussian Regularization and Convergence, New J. Phys. 23, 083004 (2021).

[113] COMSOL Inc., COMSOL Multiphysics v 5.6, https://www .comsol.com.

[114] B. Vial, F. Zolla, A. Nicolet, and M. Commandré, Quasimodal Expansion of Electromagnetic Fields in Open Two-Dimensional Structures, Phys. Rev. A 89, 023829 (2014).

[115] W. Yan, R. Faggiani, and P. Lalanne, Rigorous Modal Analysis of Plasmonic Nanoresonators, Phys. Rev. B 97, 205422 (2018).

[116] T. Christopoulos, O. Tsilipakos, O. Tsilipakos, and E. E. Kriezis, Perturbation Theory for Kerr Nonlinear Leaky Cavities, Opt. Lett. 45, 6442 (2020).

[117] J.-P. Berenger, A Perfectly Matched Layer for the Absorption of Electromagnetic Waves, J. Comput. Phys. 114, 185 (1994). 